\title{
Dynamics of a passive tracer in a velocity field of four identical point vortices
}

\author{
By STEFANELLA BOATTO $\mathrm{O}^{1,2} \dagger$ \\ AND RAYMOND T. PIERREHUMBERT ${ }^{3}$ \\ ${ }^{1}$ James Franck Institute, University of Chicago, 5640 S. Ellis Ave., Chicago, IL 60637, USA \\ ${ }^{2}$ Computational and Applied Mathematical Center, Department of Mathematics, \\ University of Chicago, 5734 S. University Ave, Chicago, IL 60637, USA \\ ${ }^{3}$ Department of Geophysical Sciences, University of Chicago, 5734 S. Ellis Ave., Chicago, \\ IL 60637, USA
}

(Received 23 April 1996 and in revised form 22 October 1998)

The dynamics of a passive tracer in the velocity field of four identical point vortices, moving under the influence of their self-induced advection, is investigated. Of interest is the change in mixing and transport properties of the tracer for the three different classes of vortex motion: periodic, quasi-periodic and chaotic. As a consequence of conservation laws, the vortex motion is confined to a finite region of phase space; therefore, the tracer phase space can be partitioned into an inner and an outer region. We find that in the case of quasi-periodic vortex motion the tracer phase space exhibits a well-defined barrier to transport between the central chaotic region and the outer region, where the trajectories are regular. In the case of chaotic vortex motion the barrier becomes permeable. The particle dynamics goes through an intermittent behaviour, where forays into the central region alternate with trapping in outer annular orbits. In the far field, an estimate of diffusion rates is made through a multipole expansion of the tracer velocity field. We make use of a specific stochastic model for the tracer velocity field which predicts no diffusion for the case of quasiperiodic vortex motion and, for the case of chaotic vortex motion, a diffusion rate that goes to zero at large distances from the vortex cluster.

\section{Introduction}

The subject of passive tracer mixing by an incompressible, unsteady flow in two dimensions has been eagerly studied, both for its intrinsic mathematical interest as a prototype for treating harder problems of fluid turbulence, and because of its direct applicability to physical problems arising in the laboratory and in nature. Large-scale atmospheric problems constitute a fruitful domain of inquiry along these lines because rapid rotation and near-conservation of entropy render the flow practically two-dimensional for many purposes. Some atmospheric applications of two-dimensional mixing concepts have been given in Sommeria, Meyers \& Swinney (1988), Pierrehumbert (1991b), and Pierrehumbert \& Yang (1993). Much attention, following Aref (1984), has focused on mixing arising from time-periodic flows. This case reduces to the problem of area-preserving maps on the plane, for which very

$\dagger$ Current address: Astronomie \& Systèmes Dynamiques, CNRS-EP 1825, Institut de Mécanique Céléste, 77 Avenue Denfert-Rochereau, 75014 Paris, France; e-mail: lella@bdl.fr. 
powerful methods of Hamiltonian chaos, such as KAM theory, can yield strong constraints on the transport properties. Strict time periodicity is not common in natural flows, and the character of mixing in such cases represents relatively unknown territory. When abandoning periodicity, it is common to resort to the other extreme of highly disordered flow, such as that arising from random maps (Chertkov et al. 1995). However, naturally occurring flows often exhibit a considerable measure of spatial and temporal order lacking in full periodicity. In the present work, we report results on a prototypical problem involving mixing due to advection by a family of chaotic flows which retain a degree of spatio-temporal structure.

Specifically, we study the mixing induced by the velocity field of four identical point vortices moving in their self-induced velocity field. This is a valid solution to the twodimensional Euler equations. The four-vortex system has a rich phenomenology, and provides a convenient source of flows that range from steadily rotating, through quasi-periodic, to slightly chaotic and on to highly chaotic (Aref \& Pomphrey 1982; Marsden \& Scheurle 1995). Additionally, the problem of mixing by concentrated vortices is interesting in its own right, because such vortices are the dominant feature of many two-dimensional turbulent flows (Carnevale et al. 1991; McWilliams 1984). Clusters of associated vortices will mix some region enclosing them. Our investigation takes some steps towards settling the following questions. How big is this region? Are there embedded unmixed islands? How big is the disk of fluid carried by each individual vortex, and how rapidly (if at all) does it mix with the larger surrounding chaotic sea? Is there some curve encircling the cluster of vortices which acts as an impermeable barrier to transport?

Although the Earth's stratosphere does not consist of a small number of pointlike vortices, its dynamics is generally controlled by a small number of irregularly vacillating planetary waves. Since the flow away from the cluster of vortices is not too sensitive to the fine details of the vorticity distribution, the problem we treat may also serve as a prototype for transport across an irregularly perturbed boundary of a stratospheric polar vortex.

Babiano and coworkers have also examined passive scalar trajectories and corresponding Lyapunov exponents in the four-vortex flow (Babiano et al. 1994). They were concerned mainly with the islands encircling the vortices when the vortices themselves undergo chaotic dynamics, and they showed how, despite the chaotic vortex motion, the dynamics of the tracer trapped in the islands is regular. $\dagger$ In this paper we will focus on the far-field dynamics for the tracer, and on how the specific vortex motion (i.e. chaotic, quasi-periodic or periodic) affects the transport properties of the tracer.

In $\S 2$ the dynamical equations, both for the vortices and for the tracer, are reviewed, with particular emphasis on the vortex dynamics. There we summarize the vortex configurations used in subsequent Sections as the basis for mixing calculations. In $\S 3$ we present some numerical results in the form of trajectories and snapshots of tracer clouds. We show that for the case of quasi-periodic vortex motion, the phase space of the tracer has a well-defined barrier to transport (referred to as the in-out barrier) separating a central chaotic region from an outer regular region where the tracer trajectories are on tori. In the case of chaotic vortex motion, the in-out barrier breaks down and a particle initially in the central region can escape, though remaining

$\dagger$ The term regular motion is used as opposed to chaotic (or irregular) motion. The degree of chaos (both for a particle and for a point vortex) is measured by investigating either the corresponding velocity spectra or the Lyapunov exponent $\lambda$ (see $\S 5$ of this paper and Lichtenberg $\&$ Lieberman 1992). 
partially trapped in ring-like orbits for long times. In $\S 4$ we discuss the statistics of finite-time Lyapunov exponents (FTLEs). Through this analysis an intermittent behaviour of the tracer can be singled out for the case of chaotic vortex dynamics. In $\S 5$ we compute a multipole expansion of the tracer velocity field in the outer field, far from the region containing the vortices. For the case of chaotic vortex motion we make use of a specific stochastic model for the velocity which predicts an asymptotically vanishing diffusion coefficient for a particle in the far field. In $\S 6$ we give some qualitative arguments about the occurrence of rings in the mixing pattern for the case of chaotic vortex motion.

Finally we remark that since the original submission of this paper an article by Wiggins \& Malhorta (1998) has also compared the difference in mixing properties of periodic, quasi-periodic and aperiodic perturbations but for a different flow: the Rossby wave flow.

\section{The dynamical equations}

The dynamics of a system of $N$ point vortices has been studied extensively in the past (Kirchhoff 1876; Aref \& Pomphrey 1982; Chorin \& Marsden 1993), and only a summary is presented here. For an unbounded domain, it is described by the following Hamiltonian system of $2 \mathrm{~N}$ equations:

$$
k_{\alpha} \dot{X}_{\alpha}=\frac{\partial H_{v}}{\partial Y_{\alpha}}, \quad k_{\alpha} \dot{Y}_{\alpha}=-\frac{\partial H_{v}}{\partial X_{\alpha}}, \quad \alpha=1, \ldots, N,
$$

with the Hamiltonian

$$
H_{v}=-\frac{1}{4 \pi} \sum_{\alpha \neq \beta} k_{\alpha} k_{\beta} \ln \left\|\boldsymbol{r}_{\alpha}-\boldsymbol{r}_{\beta}\right\|, \quad \alpha, \beta=1, \ldots, N,
$$

where $\boldsymbol{r}_{\alpha}=\left(X_{\alpha}, Y_{\alpha}\right)$ and $k_{\alpha}$ are, respectively, the coordinates and circulation of the $\alpha$ th vortex. In $(2.1),\left(X_{\alpha}, Y_{\alpha}\right)$ form a pair of canonically conjugate variables. It follows that a system of $N$ point vortices has $N$ Hamiltonian degrees of freedom and that the phase space is also the space of configurations of the system.

Our main interest is to study the dynamics of a test particle - also called a passive tracer - which is advected by the velocity field of the vortices. The tracer particle does not affect the motion of the advecting vortices and its dynamics is described by the non-autonomous Hamiltonian,

$$
H_{p}=-\frac{1}{4 \pi} \sum_{\beta=1}^{N} k_{\beta} \ln \left\|\boldsymbol{r}-\boldsymbol{r}_{\beta}\right\|,
$$

where $\boldsymbol{r}=(x, y)$ are the coordinates of the passive tracer. They can be regarded as conjugate phase-space variables. The corresponding equations of motion are

$$
\dot{x}=\frac{\partial H_{p}}{\partial y}, \quad \dot{y}=-\frac{\partial H_{p}}{\partial x} .
$$

The passive Hamiltonian $H_{p}$ depends on the vortex variables as well as on the passive tracer coordinates $\boldsymbol{r}$. This dependence of $H_{p}$ on the vortex variables leads to an explicit time dependence in that Hamiltonian:

$$
H_{p}=H_{p}\left(\boldsymbol{r}, \boldsymbol{r}_{\alpha}(t)\right)=H_{p}(\boldsymbol{r}, t) .
$$

When $H_{p}$ is time-dependent the resulting dynamics can be chaotic (Crisanti et al. 
1991). For certain vortex configurations, we therefore expect that chaotic motion might occur for the tracer. The mixing resulting from the consequent Lagrangian chaos is the principal subject of this article.

\subsection{Vortex dynamics}

Now we shall review the character of the motion of the vortices which provide the flow field for our mixing calculations. Of principal interest is determining the circumstances under which the flows are periodic, quasi-periodic or chaotic. The Hamiltonian equations of motion for the vortices can be written explicitly as

$$
\left.\begin{array}{l}
\dot{X}_{\alpha}=-\frac{1}{2 \pi} \sum_{\substack{\beta=1 \\
\alpha \neq \beta}}^{N} k_{\beta} \frac{\left(Y_{\alpha}-Y_{\beta}\right)}{\left\|\boldsymbol{r}_{\alpha}-\boldsymbol{r}_{\beta}\right\|}, \\
\dot{Y}_{\alpha}=+\frac{1}{2 \pi} \sum_{\substack{\beta=1 \\
\alpha \neq \beta}}^{N} k_{\beta} \frac{\left(X_{\alpha}-X_{\beta}\right)}{\left\|\boldsymbol{r}_{\alpha}-\boldsymbol{r}_{\beta}\right\|},
\end{array}\right\} \alpha=1, \ldots, N .
$$

In addition to the Hamiltonian $H_{v}$, a system of point vortices has the following three integrals of motion (Aref \& Pomphrey 1982):

$$
L=\sum_{\alpha=1}^{N} k_{\alpha}\left(X_{\alpha}^{2}+Y_{\alpha}^{2}\right), \quad P_{y}=\sum_{\alpha=1}^{N} k_{\alpha} Y_{\alpha}, \quad P_{x}=\sum_{\alpha=1}^{N} k_{\alpha} X_{\alpha},
$$

expressing conservation of angular and linear momentum in the plane. It can be shown that we can construct three integrals in involution $\dagger$ out of the four conserved quantities $L, P_{x}, P_{y}$ and $H_{v}$. These are $L, P_{x}^{2}+P_{y}^{2}$ and $H_{v}$. It is then possible to reduce the system of equations from $N$ to $N-2$ degrees of freedom (Aref $\&$ Pomphrey 1982). A Hamiltonian system with $N$ degrees of freedom is integrable whenever there are $N$ integrals of motion in involution + It follows that a system with $N \leqslant 3$ is integrable, whereas for four vortices or more, some additional conserved quantities are needed for this to be the case. It has been shown, however, that a system of four identical vortices, i.e. $k_{\alpha}=k$ for $\alpha=1,2,3,4$, can undergo periodic or quasi-periodic motion for special initial conditions (Aref \& Pomphrey 1982). More specifically, motion of a system of four identical vortices can be periodic, quasi-periodic or chaotic depending on the symmetry of the initial configuration.

Some typical trajectories to be considered by us are shown in figure 1 . The regular trajectories arise in the following way. In addition to being invariant under the continuous group of translations and rotations in phase space (as expected from the conservation of $P_{x}, P_{y}$ and $L$ ), the equations of motion, have a discrete symmetry. That is, if $\left\{k_{\alpha},\left(X_{\alpha}, Y_{\alpha}\right)\right\}$ is a solution, so are $\left\{k_{\alpha},\left(-X_{\alpha},-Y_{\alpha}\right)\right\}$ and $\left\{-k_{\alpha},\left(X_{\alpha},-Y_{\alpha}\right)\right\}$. These discrete symmetries have the property that, if they are satisfied by the initial configuration, they are satisfied at all subsequent times. In the specific case of a system of four identical vortices, these symmetric configurations are such that the vortices are located at the vertices of a parallelogram. We can write for that case

$$
X_{\alpha}=-X_{\alpha+2}, \quad Y_{\alpha}=-Y_{\alpha+2}, \quad \alpha=1,2 .
$$

$\dagger$ Two functions $F$ and $G$ on phase space are in involution if they Poisson-commute, $[F, G]=0$ (see Lichtenberg \& Lieberman 1992).

$\ddagger$ For an integrable system the existence of a coordinate transformation $(\boldsymbol{q}, \boldsymbol{p}) \rightarrow(\boldsymbol{I}, \boldsymbol{\theta})$ to the action angle variables $(\boldsymbol{I}, \boldsymbol{\theta})$ is proven. In these new variables the equations of motion take a quite simple form $\dot{\boldsymbol{I}}=0, \dot{\boldsymbol{\theta}}=\boldsymbol{\omega}(\boldsymbol{I})$ with corresponding solution $\boldsymbol{I}(t)=\boldsymbol{I}(0), \boldsymbol{\theta}(t)=\boldsymbol{\omega}(I) t+\boldsymbol{\theta}(0)$ (Arnold 1980). 
(a)

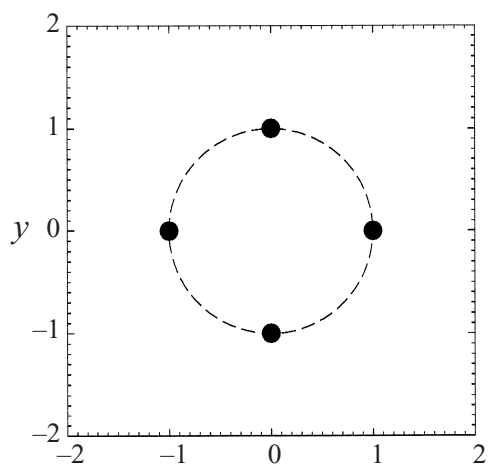

(ii)

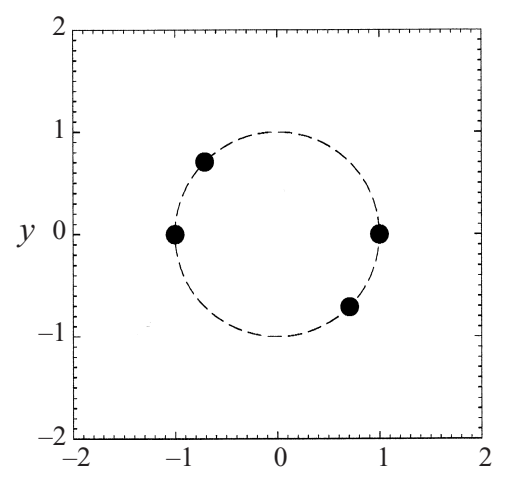

(iii)

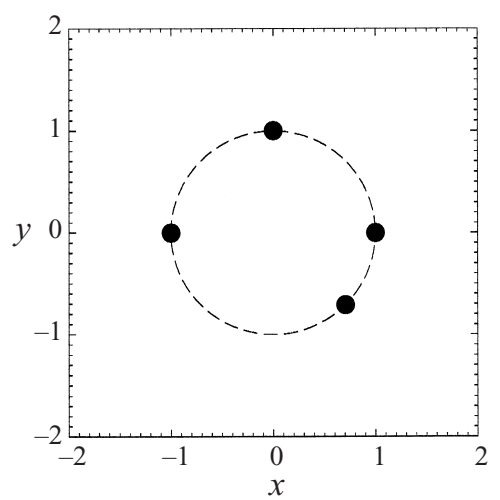

(i)
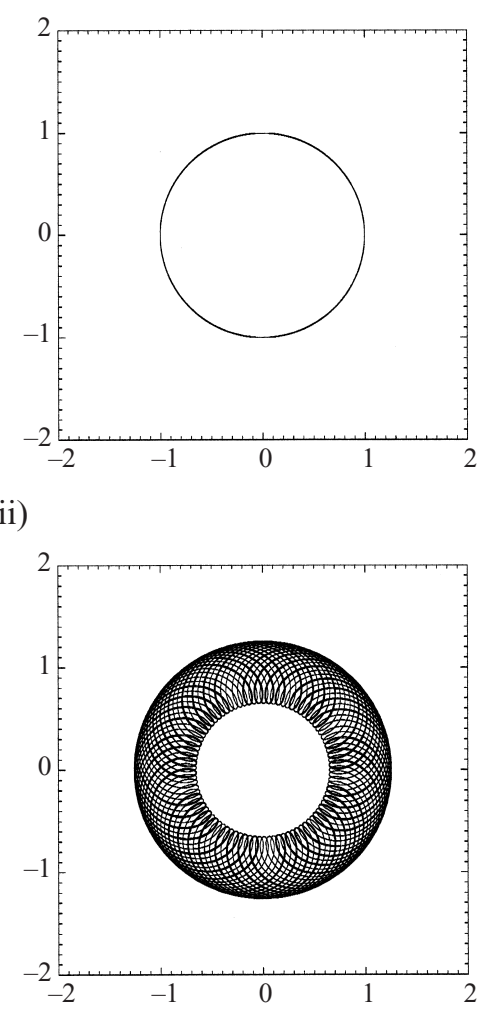

(b)

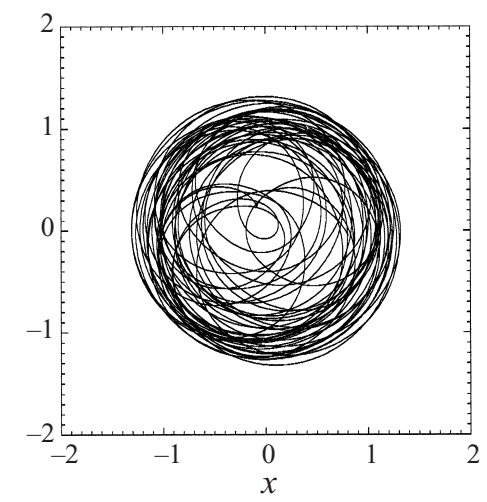

FIGURE 1. (a) Initial vortex configurations for four identical point vortices of vortex strength $k=1$. All the vortex configurations lie on a circle of radius $R=1$. (i) Square configuration, $\epsilon_{S}=0$, with energy $E_{v}=-0.882542$; (ii) $\epsilon_{S}=0.5, E_{v}=-0.661907$; (iii) $\epsilon_{A}=0.5, E_{v}=-0.747023$. (b) Corresponding trajectory for one of the vortices: (i) periodic, (ii) quasi-periodic, (iii) chaotic motion.

Whenever this equation is valid, the system can be reduced to one degree of freedom, and, due to energy conservation, the vortex motion is guaranteed to be integrable (Aref 1982, 1984; Aref \& Pomphrey 1982). The square is of particular interest because it is a stable, rotating vortex configuration. Choosing configurations that initially differ 


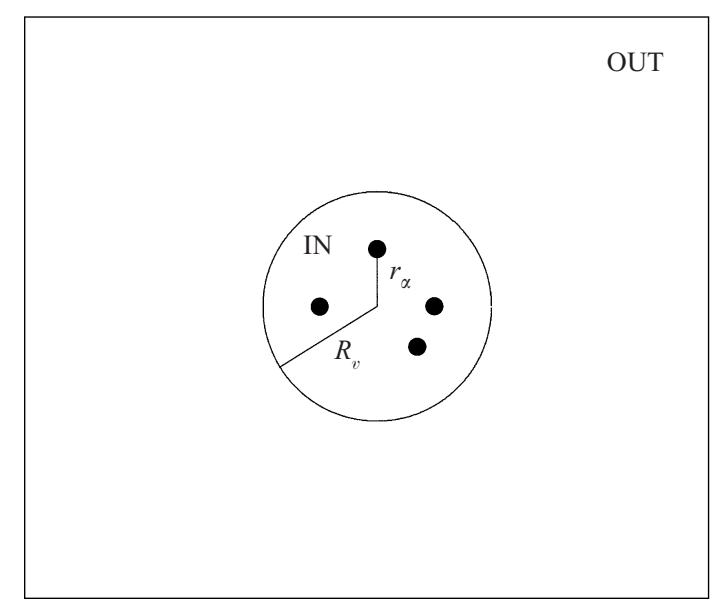

FIGURE 2. Physical space, partitioned into two distinct regions: a central disk-like region of radius $R_{v}$, IN, where the vortex motion takes place, and an outer region, OUT.

only by angular displacements offers the advantage that the angular momentum $L$ does not change. The angular momentum is of particular interest in the case of identical point vortices because it helps in estimating the size of the region where the vortex motion takes place. In fact, due to the conservation of $L=k \sum_{\alpha=1}^{N}\left(x_{\alpha}^{2}+y_{\alpha}^{2}\right)$ the vortex motion is restricted to a disk of radius $R_{v}=\sqrt{L / k}$. As a consequence of this (see §5), the tracer phase space can be thought of as partitioned into two different regions: the inner disk, denoted by IN, and its exterior, from which the vortices are excluded, denoted by OUT. See figure 2 . Without loss of generality we have chosen the units of time and distance such that $k=1$, and $L=4 . \dagger$

The vortex configurations with discrete symmetry fall into the category of integrable systems. What happens when this symmetry is broken? The vortex system is now equivalent to a system with two degrees of freedom, and chaotic vortex motion can occur. A detailed analysis of the phase-space structure of the vortices is beyond the scope of the present work. For our purposes, it will suffice to have examples to hand of quasi-periodic and chaotic four-vortex motion, without settling the general issue of when either case arises. We have found that vortex configurations with broken symmetry that are close enough to integrability display periodic or quasi-periodic dynamics. Examples include multi-clustered vortex configurations (with and without an initial symmetry) in which at least two of the vortices are close to each other and can be paired. This vortex system is equivalent to the one with two or three vortices (depending on the number of clusters), and the corresponding vortex motion is quasi-periodic.

\section{Trajectories and clouds of tracers}

In this Section we will investigate the tracer behaviour making use of numerical simulations. The basic character of the mixing will be illustrated by means of tracer trajectories and snapshots of tracer clouds. The equations for the tracer and the

$\dagger$ Note that the equations of motion can be non-dimensionalized by the time-coordinate transformations $t \rightarrow t^{\prime}=t\left(L / k^{2}\right)$ and $x_{\alpha} \rightarrow x_{\alpha}^{\prime}=x_{\alpha} \sqrt{k / L}$ since both $k$ and $L$ disappear from the equations of motion. 

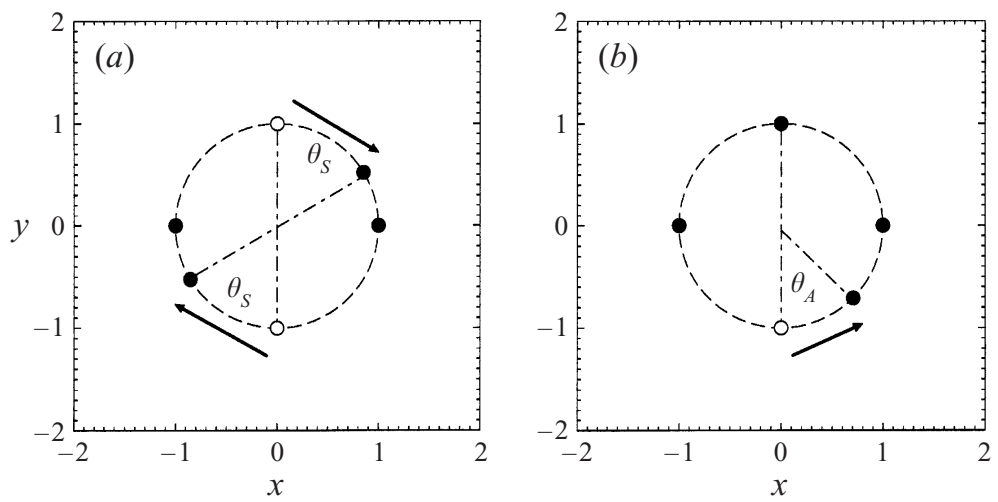

FIGURE 3 . Vortex configurations obtained by displacing $(a)$ two of the vortices by an angle $\theta_{S}=\epsilon_{S} \pi / 2$ and $(b)$ one of the vortices by an angle $\theta_{A}=\epsilon_{A} \pi / 2$, each time with respect to the square configuration.

vortices are integrated simultaneously by means of an adaptable-step-size eighth order Runge-Kutta routine. Since the validity of long-time numerical integrations is a subject of considerable interest we dedicate Appendix A to a discussion of the numerical methods employed. In our simulations, when following a particle trajectory, the position of the tracer is recorded at regular time intervals. This is done to verify where a given particle spends most of its time, to identify trapping regions for a particle by higher densities of points, and to single out barriers to transport (i.e. closed curves in the physical space which a particle cannot cross). The existence of barriers to transport can be also detected by observing the evolution of tracer clouds. Whenever a cloud of particles remains indefinitely trapped in a given region of phase space, we infer the existence of a barrier at the boundaries of such a region.

We consider symmetrical and asymmetrical vortex configurations which are perturbations of the square. We define the perturbation parameters $\epsilon_{A}$ and $\epsilon_{S}$ by means of the angular displacements, respectively, $\theta_{A}=\epsilon_{A} \pi / 2$ and $\theta_{S}=\epsilon_{S} \pi / 2$, of a single and of a pair of vortices with respect to the original square configuration, as shown in figure 3.

The square configuration is a good starting point of our analysis because the flow fields we investigate can in some sense be regarded as perturbations of the flow field of a uniformly rotating square array of identical vortices which gives rise to integrable tracer dynamics. Accordingly, an understanding of the separatrix structure of this flow considerably facilitates interpretation of the mixing results in the time-dependent case. For the unperturbed flow, it is possible by means of a coordinate transformation $(x, y) \rightarrow\left(x^{\prime}, y^{\prime}\right)$, to change to a co-rotating reference frame in which the vortices are at rest. In this new reference frame the tracer Hamiltonian is time independent:

$$
H_{p}^{\prime}=\frac{1}{2} \Omega\left(x^{\prime 2}+y^{\prime 2}\right)-\frac{k}{4 \pi} \sum_{\alpha=1}^{4} \ln \left|\boldsymbol{r}^{\prime}-\boldsymbol{r}_{\alpha}^{\prime}\right|
$$

where $\Omega=3 k / 4 \pi$. The tracer trajectories are thus identical to the streamlines, given by $H_{p}^{\prime}=$ constant. Figure 4 shows the tracer phase space in the co-rotating reference frame.

The zeros of the tracer velocity field occur only on the four symmetry axes of the vortex configuration, $x^{\prime}=0, y^{\prime}=0, x^{\prime}=y^{\prime}$ and $x^{\prime}=-y^{\prime}$. There are stable fixed 


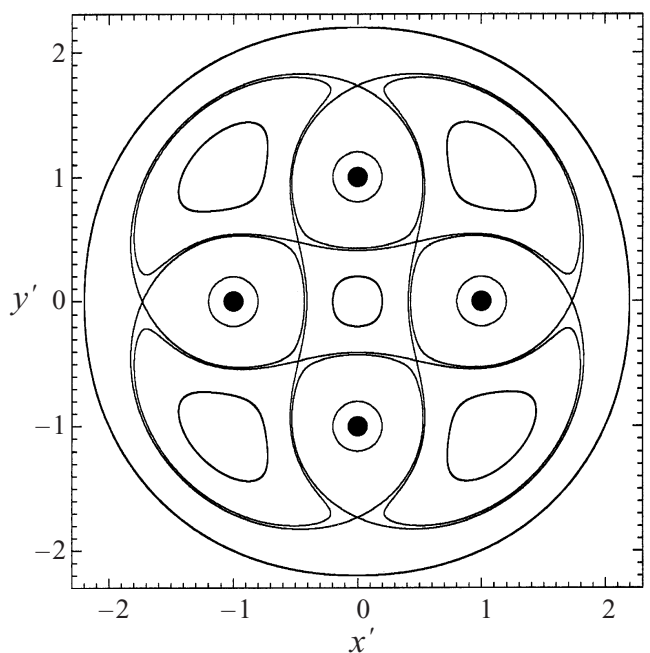

FIGURE 4. Tracer trajectories in the co-rotating vortex frame for an initial square vortex configuration. The position of a vortex is indicated by

points (elliptic points) at

$$
( \pm a, \pm a) \text { and }(0,0)
$$

and unstable fixed points (hyperbolic points) at

$$
( \pm b, \pm b),(0, \pm \sqrt{3}) \text { and }( \pm \sqrt{3}, 0)
$$

where $a=\sqrt{(4+\sqrt{7}) / 6}$ and $b=\sqrt{(4-\sqrt{7}) / 6}$. Since the flow is stationary, if the stable and unstable manifolds of hyperbolic fixed points intersect once, they intersect at every point (i.e. no transversal intersections can occur) (see Lichtenberg \& Lieberman 1992). Those separatrices form a net of eight homoclinic and eight heteroclinic orbits, partitioning phase space into a group of lobes forming a central region and an outer region in which the orbits encircle the central region. Notice that the separatrices can come quite close without touching. The outer family of orbits forms a circular boundary separating the inner vortex-containing region from an outer region filled with topologically circular orbits. There is also the expected isolated family of nearly circular orbits encircling each point vortex. Less obviously, there are a number of isolated lobes in the inner region, which do not contain a vortex.

When the initial square vortex configuration is perturbed by some small displacement of the vortices, a time dependence is introduced into the tracer Hamiltonian. The vortex motion is either quasi-periodic or chaotic, and there is no longer, for the general case, a co-rotating frame in which the vortices are at rest. The flow is no longer stationary and transversal intersections of stable and unstable manifolds of given fixed points may occur (Wiggins 1991). Transport of particles across the separatrices will occur, leading to the formation of a stochastic layer surrounding islands of stable orbits (Rom-Kedar 1993). Particle transport takes place within the area of the stochastic sea and corresponds to a wandering amidst the islands.

\subsection{Trajectories of tracers}

In figure 5 two trajectories of a tracer initially in the inner region are shown. These are for two different kinds of vortex motion: quasi-periodic $\left(\epsilon_{s}=0.1\right)$ and chaotic $\left(\epsilon_{A}=0.5\right)$. 
(a)
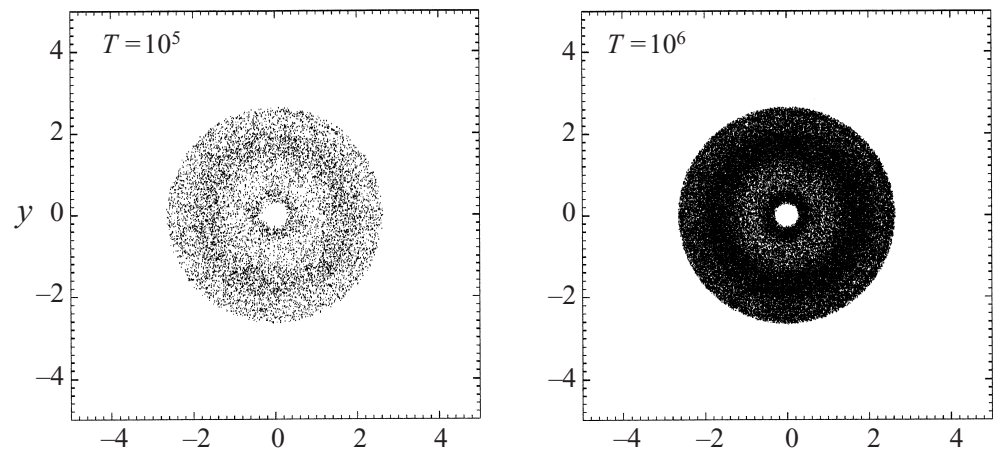

(b)
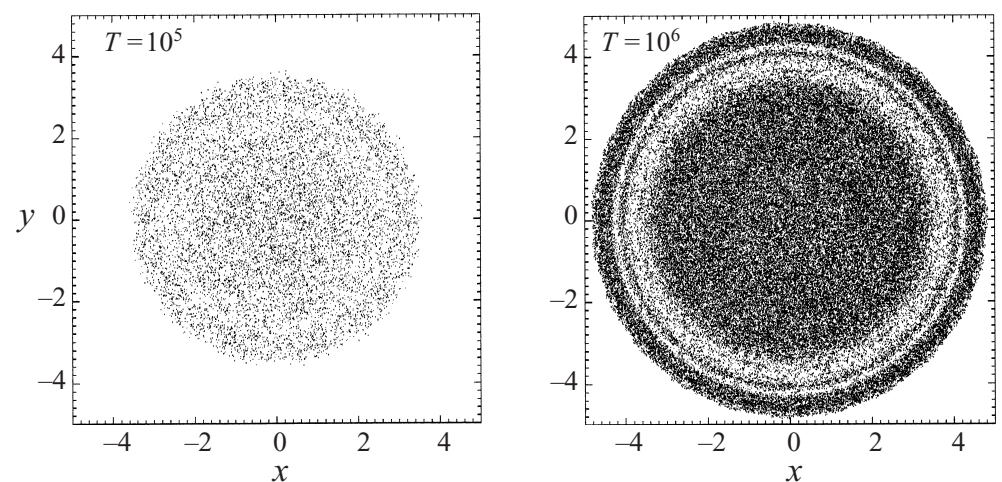

FIGURE 5. Trajectories of particles initially in the inner region. (a) Quasi-periodic vortex motion $\left(\epsilon_{s}=0.1\right)$ : a particle trajectory remains confined in the central disk-like region even when longer times $\left(T_{2}=2 \times 10^{6}\right)$ are considered. (b) Chaotic vortex motion $\left(\epsilon_{A}=0.5\right)$ : initially a particle trajectory fills a disk-like region, then, for long enough times, it will leave the disk-like region and be trapped in ring-like orbits.

For quasi-periodic vortex motion particle trajectories show chaotic behaviour and remain confined to the central region for long times. The sense in which these trajectories are chaotic will be made more precise in $\S \S 3.2$ and 4 . This behaviour suggests the existence of a barrier (the in-out barrier) to transport between the inner and the outer regions. The existence of such a barrier is further confirmed when looking at trajectories of particles initially in the outer region (see figure 6). The trajectories for outer particles are found to be predominantly regular (they are quasiperiodic), occupying an annulus-like region whose width, $W_{A}=r_{\max }-r_{\min }$, decreases with the radial distance (see Boatto 1996).

The tracer is much less constrained in the case of chaotic vortex motion. A particle initially in the inner region is only temporarily confined there. For long enough times it can enter the outer region, where it diffuses slowly through the radial coordinate, occupying, for intermediate time scales, ring-like orbits (see figure 7). As will be discussed also in $\S 4$, the radial diffusion exhibits an intermittent behaviour, where episodes of trapping in the rings alternate with flights in the central chaotic sea. Therefore the in-out barrier becomes semi-permeable in the case of chaotic vortex motion. The process of entering and exiting the central region seems to repeat 

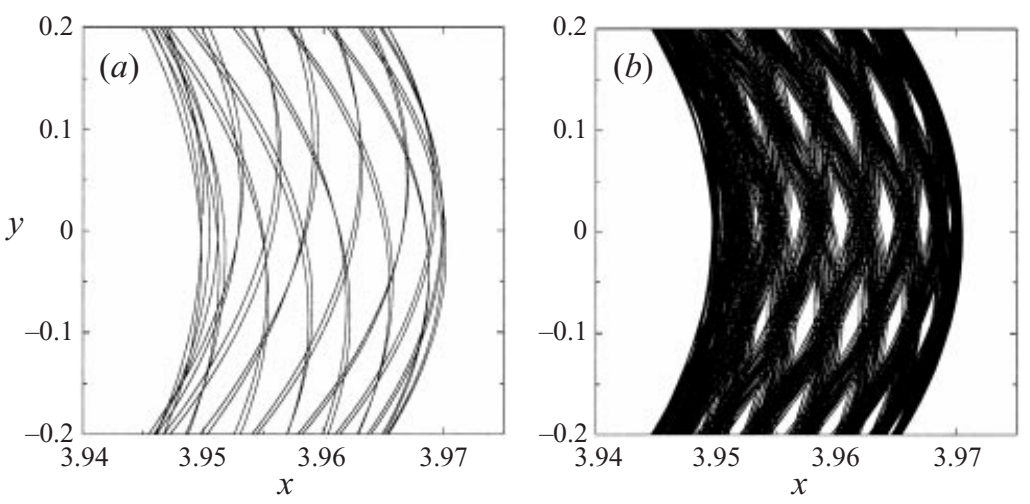

FIGURE 6. Trajectory of a particle initially in the outer region for quasi-periodic vortex motion $\left(\epsilon_{S}=0.1, E_{v}=-0.874656\right)$. Close-ups of the trajectory at $(a) T_{1}=5000$ and $(b) T_{2}=10^{5}$. The tracer trajectory is a torus-like orbit which occupies an annular region of width $W_{A}=r_{\max }-r_{\min }$.

ad infinitum and the following questions arise: Can a particle eventually escape to infinity? Does a barrier (or equivalently a critical radius $R_{c}$ ) exist beyond which particles cannot enter the central region? Why does a well-defined in-out barrier exist in the case of regular vortex dynamics?

In the case of chaotic vortex motion and tracers whose initial radius $R_{o}$ is much greater than the vortex radius $R_{v}=\sqrt{L / k}$, the diffusion time is necessarily quite long, owing to the rapid decay of the fluctuating radial velocity with $R_{o}$. An analytical estimate of the diffusion rate will be developed in $\S 5$.

\subsection{Temporal sequences of clouds of tracers}

Trajectories give only a blurry picture of the phase-space structure, since they represent a two-dimensional projection of a higher-dimensional entity. Instead of plotting the trajectory of a single particle, we can lay down a localized cloud of independent tracer particles initially, and plot snapshots of the positions of the particles at selected later times. This unambiguously maps out the connected mixing region that contains the initial cloud, and also distinguishes chaotic regions (where the cloud fills a twodimensional area) from invariant tori.

For the case of quasi-periodic vortex motion we consider initial vortex configurations which are symmetrical angular perturbations of the square. In figure 8 we compare the evolution of tracer clouds for the different vortex configurations considered. We notice that for small angular perturbations, the separatrices are replaced by a thin stochastic layer which surrounds islands of stable non-random orbits (figure $8 a$ ). So in this case the topology of the tracer phase space is very close to the one induced by the square vortex configuration (figure 4). When the angular perturbation is increased (figure $8 b-d$ ) some of the regular islands split and stretch. During their wanderings, particles can get trapped, for relatively long times, at the boundaries of the islands by semi-permeable (partial) barriers, also called cantori (Percival 1979; Montroll \& Schlesinger 1984; MacKay, Meiss \& Percival 1987). This partial trapping of a particle in the neighbourhood of a regular island is known in literature as the stickiness phenomenon, and it manifests itself in our simulation by a higher density of points near some of the islands, as shown in figure $8(d)$. Notice that when $\epsilon_{s}$ approaches unity, the configuration reduces to a pair of vortex clusters and the flow field is accurately described as a perturbation of a vortex pair, of effective circulation 

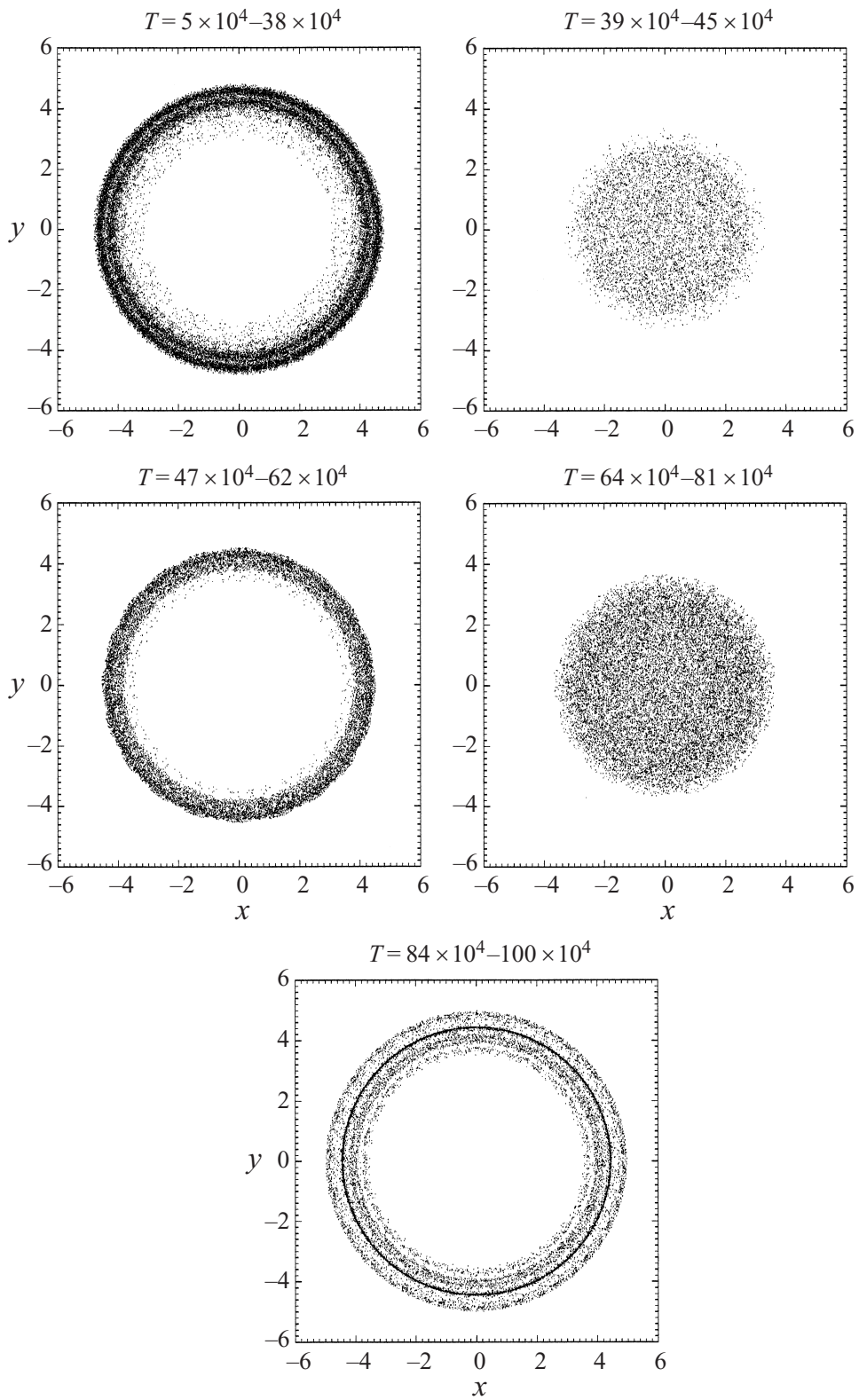

FIGURE 7. Intermittent behaviour of a tracer trajectory in a case of chaotic vortex motion $\left(\epsilon_{A}=0.5\right)$. Confinement to the central chaotic region alternates with trappings in the outer annular orbits.

$K=2 k$. The familiar separatrix structure of this case consists of a simple pair of homoclinic orbits, forming a figure-eight joined at the midpoint between the two vortices (see figure $8 e, f$ ).

To show the dynamics, in figure 9 a temporal sequence for a tracer cloud is shown, for the case $\epsilon_{s}=0.01$. The cloud is initially stretched, then folded and stretched again; by the end, after some characteristic mixing time, the cloud is uniformly spread over the chaotic region. Notice that even after relatively long times the boundaries of the region occupied by the cloud remain sharp and well defined, hinting at the existence of 

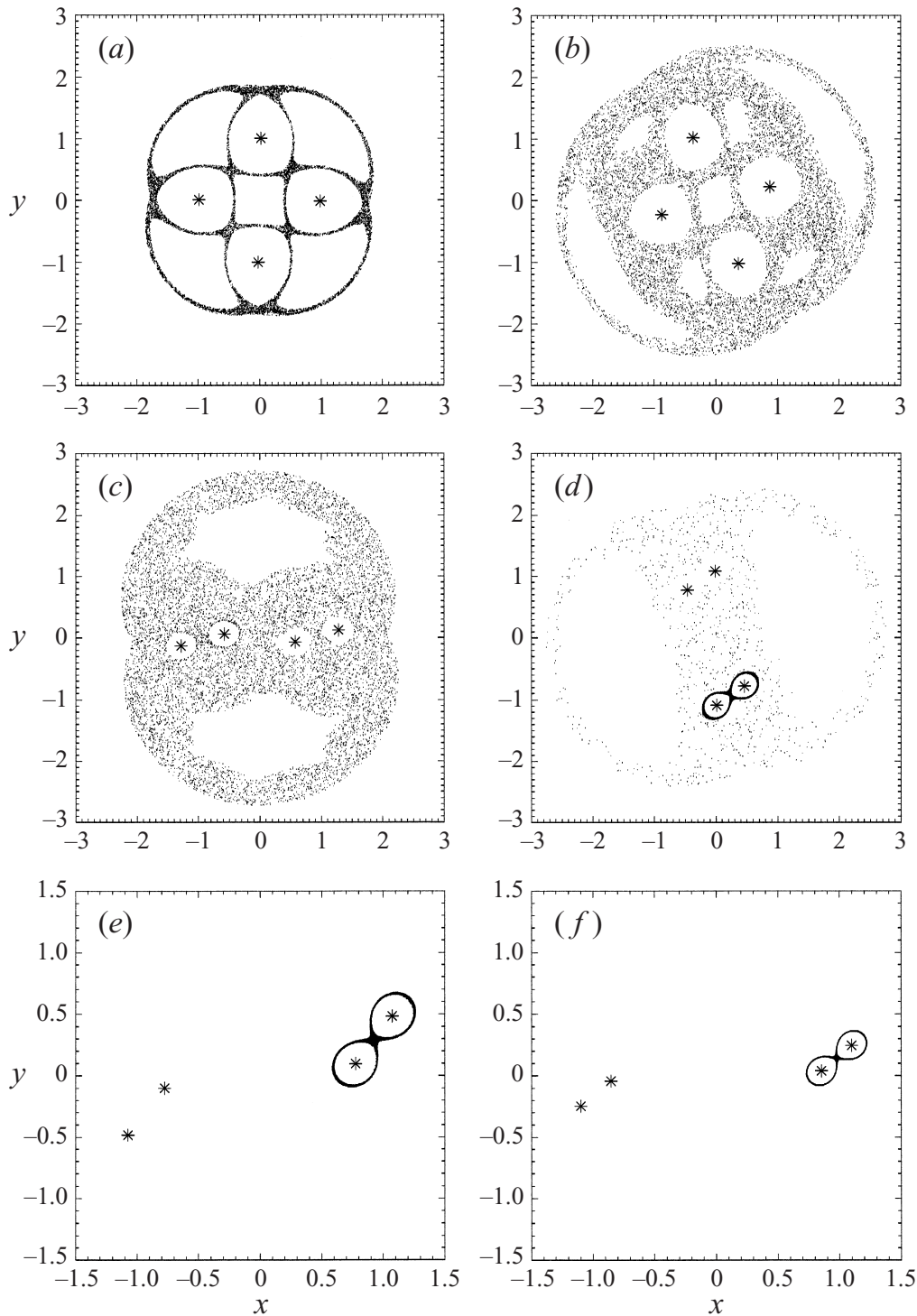

FIGURE 8. Tracer clouds for different, symmetric initial vortex configurations (i.e. quasi-periodic vortex motion, and $\theta_{S}=\epsilon_{S} \pi / 2$ ). For all cases we have chosen, as inital condition for the tracers, a cloud of particles concentrated near the middle point of a pair of close vortices, to show how, as the vortices get closer (increasing $\epsilon_{S}$ ), the resulting stochastic region approaches the separatrix of an isolated vortex pair. (a) $\epsilon_{s}=0.01, E_{v}=-0.882464 ;(b) \epsilon_{s}=0.1, E_{v}=-0.874656 ;(c) \epsilon_{s}=0.6$, $E_{v}=-0.544247 ;(d) \epsilon_{s}=0.65, E_{v}=-0.469291 ;(e) \epsilon_{s}=0.7, E_{v}=-0.379817 ;(f) \epsilon_{s}=0.8$ $E_{v}=-0.134922$, where $E_{v}$ is the value of the Hamiltonian of the vortices. The position of a vortex is indicated by $*$.

barriers. In particular one can distinguish between an external barrier, separating the central chaotic region from the outer regular one, and internal barriers, demarcating the inner regular islands. Of the inner regular islands, four surround the vortices, one encircles the origin (which is a stable fixed point for the flow) and the remaining four are related to four elliptic fixed points of the flow. 
(a) $T=60$

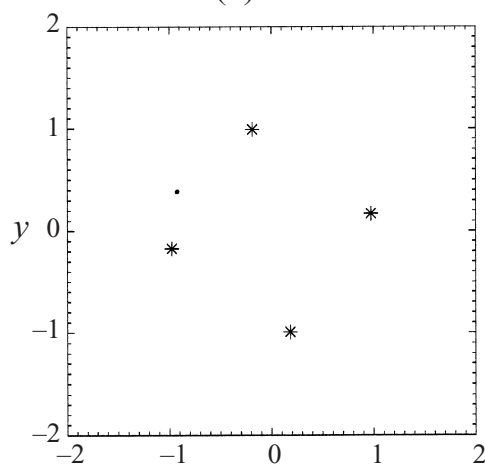

(c) $T=250$

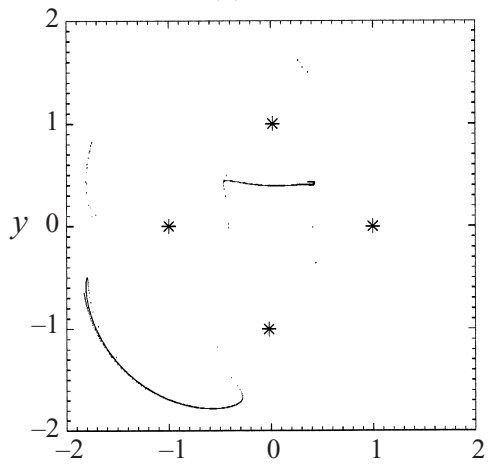

(e) $T=350$

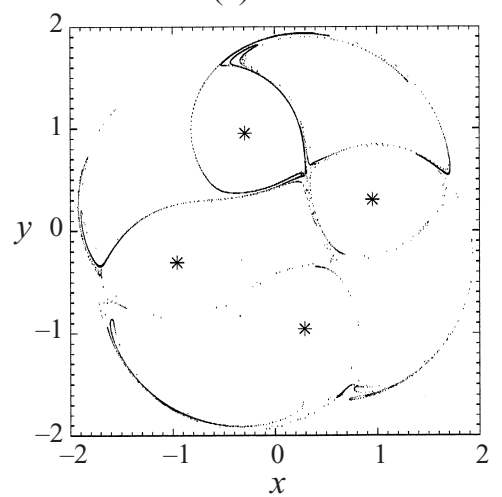

(b) $T=200$

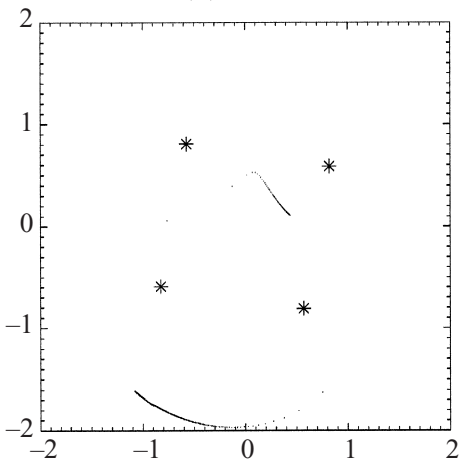

(d) $T=300$

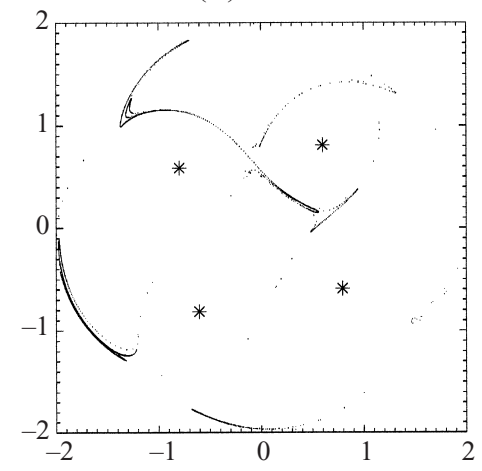

(f) $T=1000$

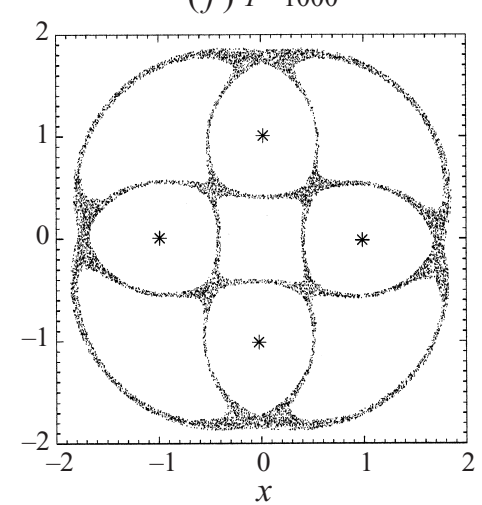

FIGURE 9. Temporal sequence for a cloud of tracers in a case of regular vortex motion. The corresponding initial vortex configuration is symmetric with an angular displacement $\theta_{S}=0.01 \pi / 2$ (or equivalently with $\epsilon_{S}=0.01$ ). The position of a vortex is indicated by $*$.

In figure 10 a temporal sequence for a tracer cloud advected by chaotic vortex motion is shown $\left(\epsilon_{A}=0.5\right)$. The advecting field is generated by the chaotic vortex motion shown in figure 1. The particle cloud stretches and folds up to fill the central chaotic region. Regular islands encircling the vortices are still preserved, as for the case of quasi-periodic vortex motion. Consistent with Babiano et al. (1994), we find that these appear to be the only unmixed islands surviving in the inner region, 
(a) $T=30$

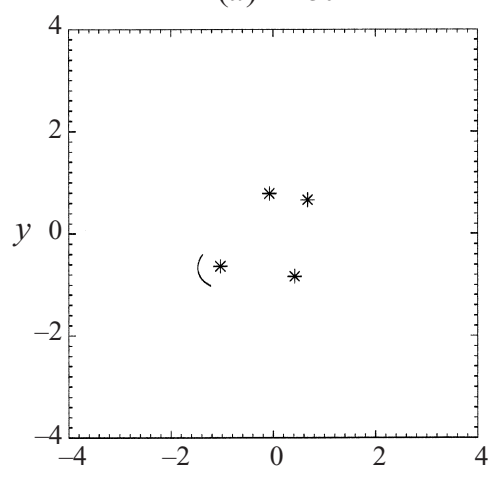

(c) $T=100$

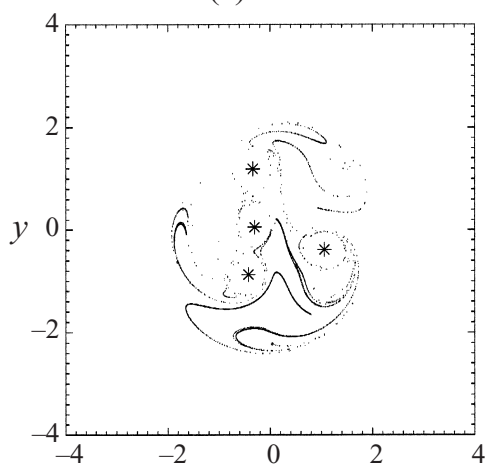

(e) $T=600$

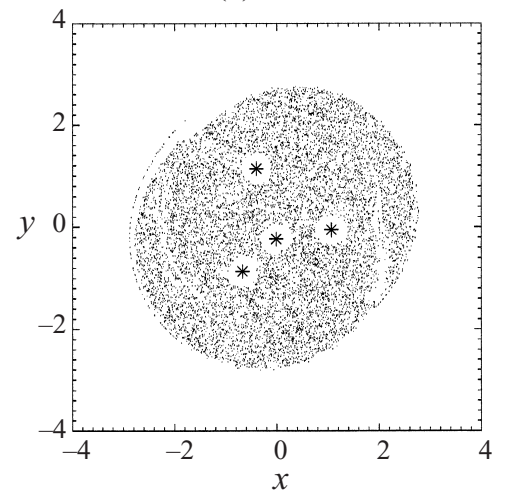

(b) $T=60$

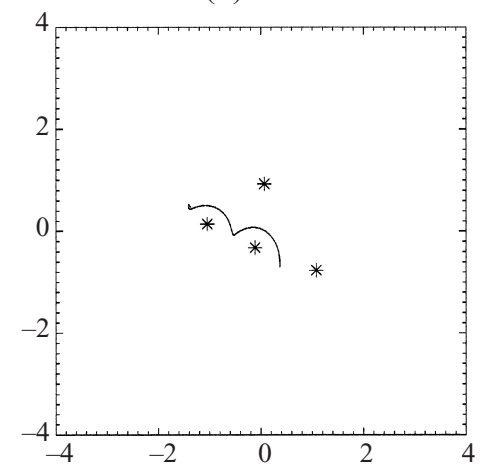

(d) $T=200$

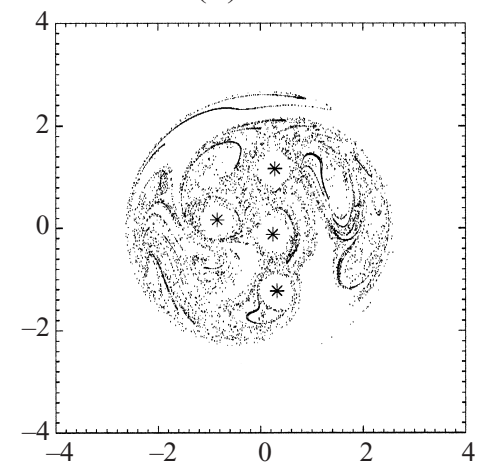

(f) $T=700$

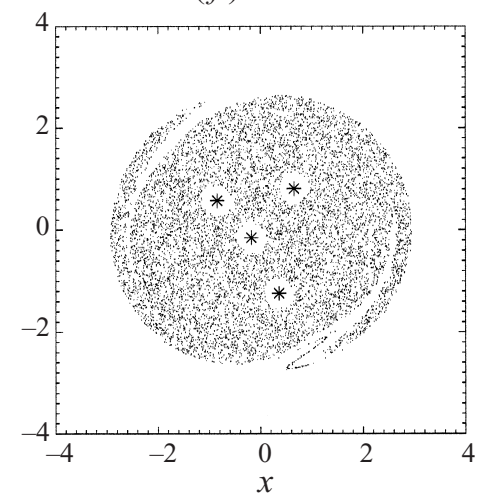

FigurE 10. Temporal sequence for a cloud of tracers in a case of chaotic vortex dynamics. The corresponding initial vortex configuration is asymmetric, with an angular displacement $\theta_{A}=0.5 \pi / 2$ (or equivalently with an $\epsilon_{A}=0.5$ ). The position of a vortex is indicated by $*$.

and correspond to rapidly circulating, nearly circular, regions of fluid carried along with the vortices. In contrast to the case of quasi-periodic dynamics, we observe no additional macroscopic island of regularity. For long enough times, some of the tracer particles exit the central region (figure 10), defining spiral-like filaments, and occupying ring-like regions (see figure $10 e, f$ ). As already seen in the trajectory analysis (figure 5), chaos in the advecting field breaks the in-out barrier, allowing formerly confined particles to leak out. 
(a)

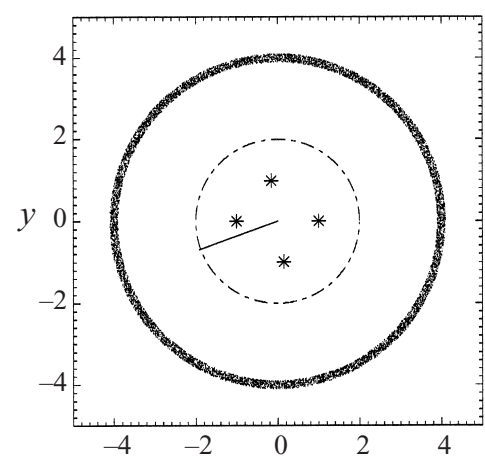

(b)
$T=0$

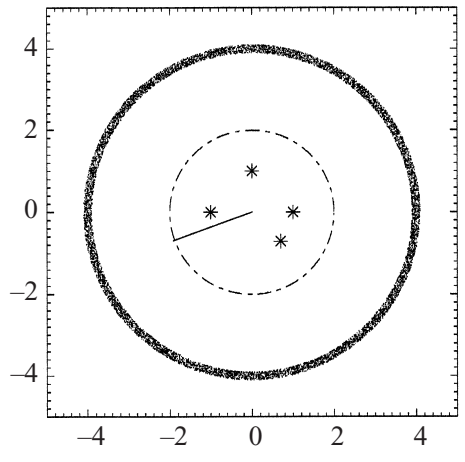

$T=4000$
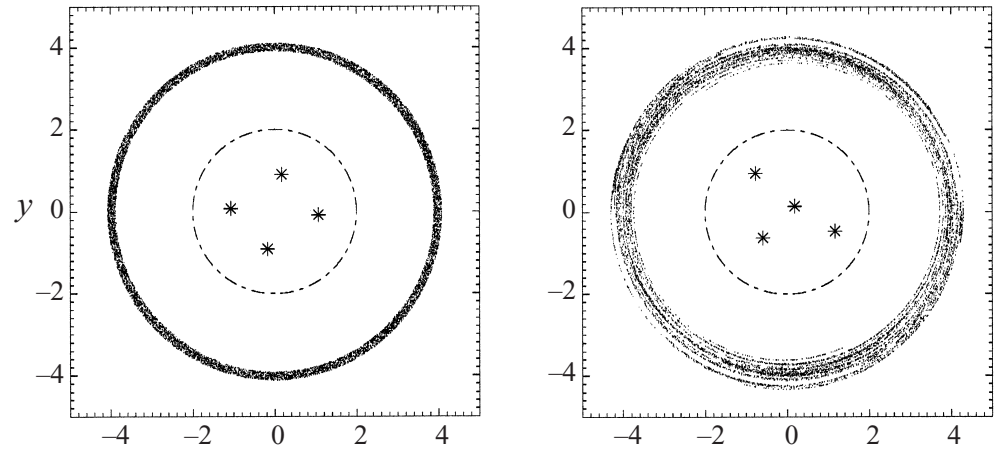

$T=10^{4}$
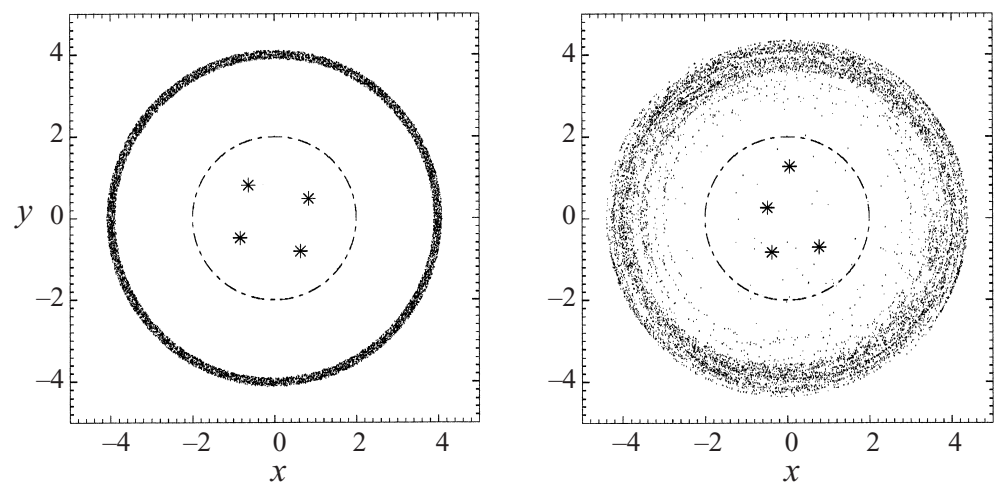

FIGURE 11. Time evolution of a cloud of tracers, initially distributed uniformly over a thin annulus centered at $R=4$ in the outer region. (a) Quasi-periodic vortex dynamics $\left(\epsilon_{S}=0.1\right) ;(b)$ chaotic vortex motion $\left(\epsilon_{A}=0.5\right)$. The position of a vortex is indicated by $*$.

The structure of the outer region is further explored by considering a cloud of particles distributed uniformly over a thin annular region, as shown in figure 11 . The difference between a quasi-periodic and a chaotic advecting vortex motion is quite striking. In the case of quasi-periodic vortex motion the tracers remain confined to a well-defined annular region, i.e. no diffusion occurs (see discussion in §5.2). 
In the case of chaotic vortex motion, after some initial time, the tracers are preferentially located on some ring-like regions, as shown in figure 11(b). The cloud of particles is not confined to a fixed annular region and diffusion can occur, as will be confirmed in $\S 5.2$, by means of both a numerical estimate and a stochastic model.

\section{Finite-time Lyapunov exponent analysis}

The study of Lyapunov exponents (LE) is an important guide for understanding the mixing properties and the degree of chaoticity of a fluid (Pierrehumbert 1991b). Integrable systems are characterized by zero or negative Lyapunov exponents (Bettin et al. 1980; Oseledec 1968), and mixing is generally suppressed, while chaotic systems are characterized by positive Lyapunov exponents, and mixing is generally favoured. There are several additional reasons why stretching analysis has received so much attention. First, even when we can make use of Poincare sections, as in the case of periodic flows, the standard trajectory plots often fail to capture important structures, as shown by Muzzio, Swanson \& Ottino (1991) for a particular globally chaotic flow. However, these structures manifest themselves quite clearly, when using the evolution of stretching and stirring. Secondly, when studying aperiodic flows, we cannot make use of most of the tools commonly used to analyse chaotic mixing in periodic flows - such as Poincare sections, periodic points and their associated manifolds. Hence other approaches are required to explore the phase space of the system. Finite-time Lyapunov analysis of the flow will be our third diagnostic method, in addition to particle tracking and cloud spreading.

\subsection{Definition of Lyapunov exponents}

Given an $M$-dimensional flow $\boldsymbol{y}(t)$ generated by

$$
\frac{\mathrm{d} y_{i}}{\mathrm{~d} t}=F_{i}(\boldsymbol{y}, t), \quad i=1,2, \ldots, M,
$$

the Lyapunov exponent for an initial point $\boldsymbol{y}_{o}$ is defined by the exponential rate of expansion of a displacement vector $\xi(t)$ which evolves according to the linearized equation of motion

$$
\frac{\mathrm{d} \xi}{\mathrm{d} t}=\left.\frac{\partial \boldsymbol{F}}{\partial \boldsymbol{y}}\right|_{\boldsymbol{y}(t)} \cdot \boldsymbol{\xi}(t)
$$

Here $\boldsymbol{y}(t)$ is the trajectory starting at $\boldsymbol{y}(0)=\boldsymbol{y}_{o}$ and $\boldsymbol{\xi}(t)$ approximates the displacement between two close trajectories $\boldsymbol{y}_{1}, \boldsymbol{y}_{2}$ with $\boldsymbol{y}_{1}(0)=\boldsymbol{y}_{0}$ and $\boldsymbol{y}_{2}(0)=\boldsymbol{y}_{1}(0)+\boldsymbol{\xi}(0)$. The Lyapunov exponent is defined by

$$
\lambda\left(\boldsymbol{y}_{o}\right)=\lim _{t \rightarrow \infty} \lim _{D\left(\boldsymbol{y}_{o}, 0\right) \rightarrow 0} \frac{1}{t} \ln \frac{\mathrm{D}\left(\boldsymbol{y}_{o}, t\right)}{\mathrm{D}\left(\boldsymbol{y}_{o}, 0\right)}=\lim _{t \rightarrow \infty} \frac{1}{t} \ln \frac{\mathrm{d}\left(\boldsymbol{y}_{o}, t\right)}{\mathrm{d}\left(\boldsymbol{y}_{o}, 0\right)}
$$

where $\mathrm{D}\left(\boldsymbol{y}_{o}, t\right)=\left\|\boldsymbol{y}_{2}(t)-\boldsymbol{y}_{1}(t)\right\|$ and $\mathrm{d}\left(\boldsymbol{y}_{o}, t\right)=\left\|\boldsymbol{\xi}\left(\boldsymbol{y}_{o}, t\right)\right\|$. In numerical calculations we cannot approach the infinite time limit and, in addition, for most of the systems of physical relevance we are interest in finite-time processes (Chertkov et al. 1995): we must then work with finite-time Lyapunov exponents (FTLEs). It turns out that the distribution of FTLEs contain a great deal of information about the topology of the region under consideration, such as, for example, the existence of KAM tori and semi-permeable barriers to transport. When the infinite $t$ limit is taken, almost all initial $\boldsymbol{y}_{o}$ in the same connected region of space, say $C$, share the same Lyapunov exponent, $\lambda\left(\boldsymbol{y}_{o}\right)=\lambda_{C}$ (Lichtenberg \& Lieberman 1992). Each region of the space is 
then characterized by a unique Lyapunov exponent. The scenario is quite different when we consider FTLE instead. A finite-time Lyapunov exponent is defined by

$$
\lambda\left(y_{o}, T\right)=\frac{1}{T} \ln \frac{\mathrm{d}\left(\boldsymbol{y}_{o}, T\right)}{\mathrm{d}\left(\boldsymbol{y}_{o}, 0\right)} .
$$

This slowly converges to the corresponding Lyapunov exponent as $T \rightarrow \infty$, but points in the same region might have significantly different FTLEs for moderate times $T$. Therefore a given region $C$ is characterized by a distribution of FTLEs $\left\{\lambda\left(\boldsymbol{r}_{o}, T\right)\right\}_{\boldsymbol{r}_{o} \in C}$ rather than just a single value $\lambda_{C}$. For our particular model there is a distinction to be made between the tracer variables, $\boldsymbol{r}=(x, y)$, and the field variables, $\boldsymbol{r}_{\alpha}=\left(X_{\alpha}, Y_{\alpha}\right)$ with $\alpha=1,2,3,4$. For LEs we will focus only on the two-dimensional tracer phase subspace. Write the evolution equations as

$$
\frac{\mathrm{d} \boldsymbol{r}}{\mathrm{d} t}=\boldsymbol{V}(\boldsymbol{r}, t), \quad V_{i}(\boldsymbol{r}, t)=F_{i}\left(\boldsymbol{r}, \boldsymbol{r}_{\alpha}(t)\right) \quad i=1,2, \quad \alpha=1, \ldots, 4,
$$

where $\boldsymbol{V}(\boldsymbol{r}, t)$ is a vector in the two-dimensional tracer phase space, the Eulerian velocity field. The corresponding linearized equation (the analogue of (4.2)) is

$$
\frac{\mathrm{d} \xi}{\mathrm{d} t}=\mathbf{J}(t) \cdot \xi
$$

where $\boldsymbol{\xi}(0)=\left(\left(x_{2}-x_{1}\right)(0),\left(y_{2}-y_{1}\right)(0)\right)$ and $\boldsymbol{J}=\left.(\partial \boldsymbol{V} / \partial \boldsymbol{r})\right|_{\boldsymbol{r}(t)}$. In this subspace it is possible to introduce the mean exponential rate of divergence, or tracer Lyapunov exponent (Crisanti et al. 1991), for two trajectories that are initially close in the same way as we do for the whole system (4.3):

$$
\lambda_{T r}\left(\boldsymbol{r}_{o}\right)=\lim _{t \rightarrow \infty} \frac{1}{t} \ln \frac{\mathrm{d}\left(\boldsymbol{r}_{o}, t\right)}{\mathrm{d}\left(\boldsymbol{r}_{o}, 0\right)},
$$

Notice that this depends on the initial vortex configuration $\left(\left\{\boldsymbol{r}_{\alpha}(0)\right\}\right)$ as well as tracer position but that we measure distances $\mathrm{d}\left(\boldsymbol{r}_{o}, t\right)$ only with respect to the tracer variables. We have Lagrangian chaos whenever the solution of (4.5) has a sensitive dependence on initial conditions, i.e. initially close trajectories diverge exponentially fast. We quantify this notion by saying that the Lagrangian Lyapunov exponent in (4.7) is positive (Crisanti et al. 1991; Lichtenberg \& Lieberman 1992).

The equations of motion for the tracer are Hamiltonian and therefore the LEs and FTLEs occur in reciprocal pairs $\lambda_{T r 1}\left(\boldsymbol{r}_{o}\right)=-\lambda_{T r 2}\left(\boldsymbol{r}_{o}\right)$. This means that there is one contracting and one diverging stretching direction and generically an arbitrarily chosen initial displacement will be predominantly aligned along the diverging direction after some time. Regular motion is expected when both eigenvalues are identically zero. Since we will be focusing on the tracer behaviour, from now on we will use the notation $\lambda(\boldsymbol{r})$ in place of $\lambda_{T r}(\boldsymbol{r})$.

\subsection{Numerical estimates}

There are several numerical schemes to compute the FTLE. In this paper we will employ an algorithm that makes direct use of the solutions of the linearized system, (4.6). Defining $\mathrm{d}\left(\boldsymbol{r}_{o}, t\right)$ as we did after (4.7) and plotting $\ln \left(\mathrm{d}\left(\boldsymbol{r}_{o}, t\right) / \mathrm{d}\left(\boldsymbol{r}_{o}, 0\right)\right)$ versus time, one can evaluate the FTLE as the asymptotic slope of the curve $F\left(\boldsymbol{r}_{o}, t\right)=$ $\ln \left(\mathrm{d}\left(\boldsymbol{r}_{o}, t\right) / \mathrm{d}\left(\boldsymbol{r}_{o}, 0\right)\right)$. It should be mentioned that another widely used method of estimating the FTLEs is, for small enough sampling time $\Delta \tau$, the average over the largest eigenvalues of the Jacobian of the flow, $\boldsymbol{J}$ (4.6) (Ott 1993; Horita et al. 1990; 
(a)

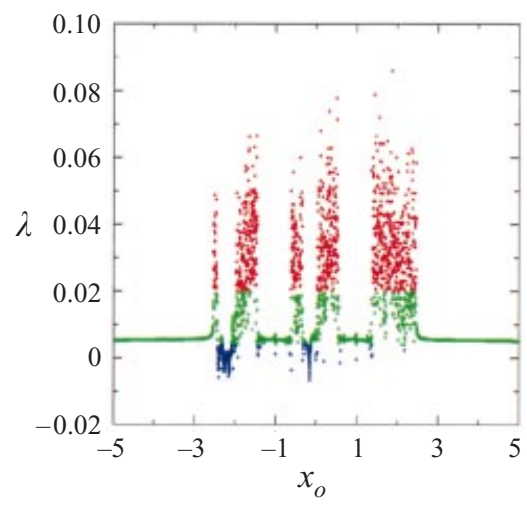

$T=500$

(b)

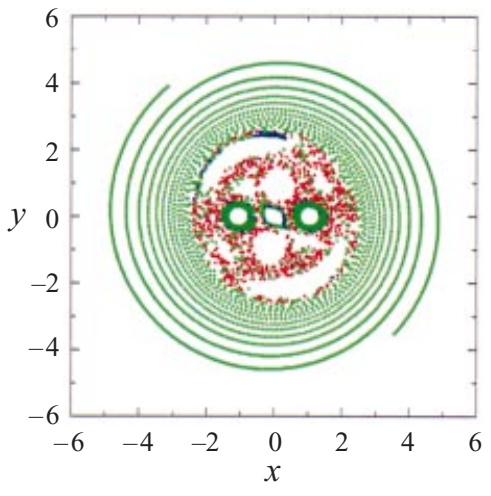

$T=4000$
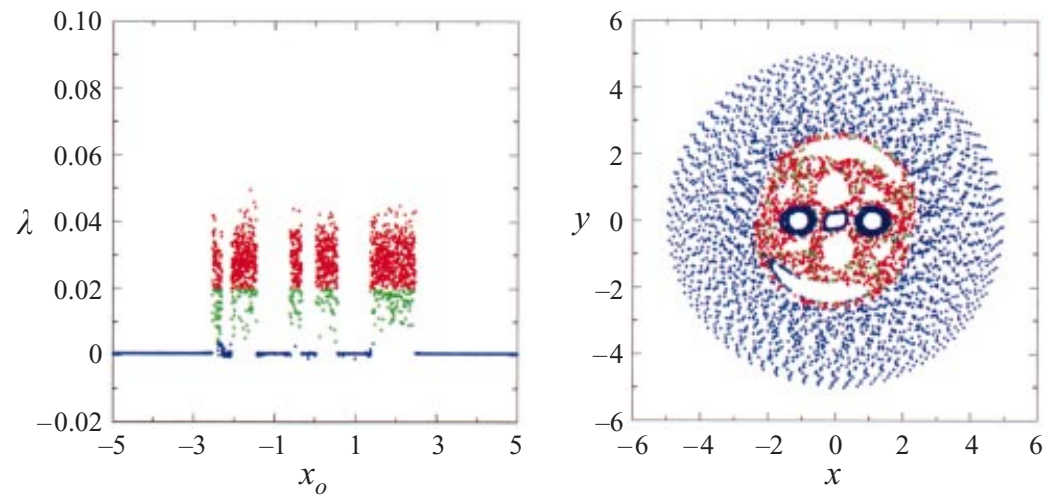

FIGURE 12. $\epsilon_{S}=0.1$. (a) Plots of finite-time Lyapunov exponents, $\lambda(\boldsymbol{r}, T)$, with respect to the initial location, $\boldsymbol{r}=(x, C)$, of the particles on a horizontal line $(y=C)$, in a quasi-periodic vortex dynamics at finite times $T_{1}=500$ and $T_{2}=4000$. The values of $\lambda(r, T)$ have been divided into three ranges, which are indicated by different colours: red, green, and blue respectively indicate high stretching $(\lambda>0.02)$, medium stretching $(0.004<\lambda<0.02)$, and low stretching $(\lambda<0.004)$. (b) Snapshots of the tracer cloud at $T_{1}=500$ and $T_{2}=4000$. Particles have been colour-coded by the corresponding finite-time Lyapunov exponent.

Badii \& Politi 1987), namely

$$
\lambda\left(\boldsymbol{r}_{o}, T\right)=\frac{1}{T} \sum_{n=1}^{N} \lambda\left(t_{n}\right)^{+} \Delta \tau,
$$

where $T$ is the total elapsed time, $N$ is the number of time steps in the time interval $T$ (i.e. $T=N \Delta \tau$ ), and $\lambda\left(t_{n}\right)^{+}$is the largest eigenvalue of the Jacobian of the flow, $J$, (4.6), at the time $t_{n}=n \Delta \tau$.

This second method of estimating FTLE is equivalent to keeping the linearized vector $\boldsymbol{\xi}\left(\boldsymbol{r}_{o}, t\right)$ always aligned along the direction of maximum stretching. This has only proven to be valid for some systems that are strictly hyperbolic (Horita et al. 1990), and is not guaranteed to work in cases where KAM tori exist. We illustrate this point in Appendix B with a particular non-hyperbolic system, where we show that algorithm (4.8) gives a wrong answer for the tracer dynamics. This is because fluctuations of the strain direction are not taken into account properly. 
(a)

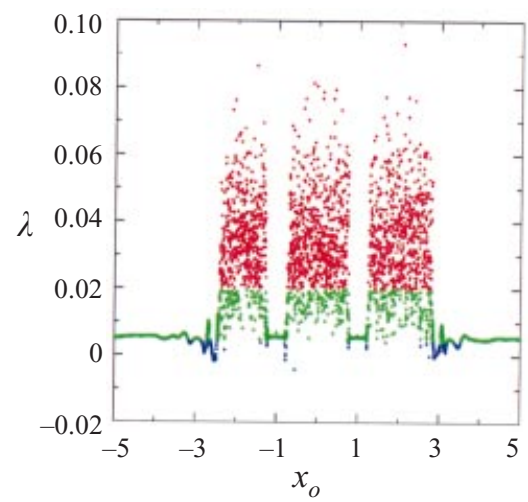

$T=500$

$T=4000$

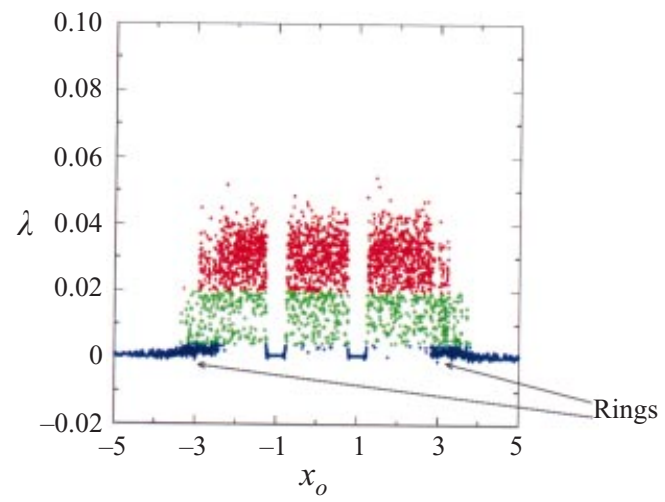

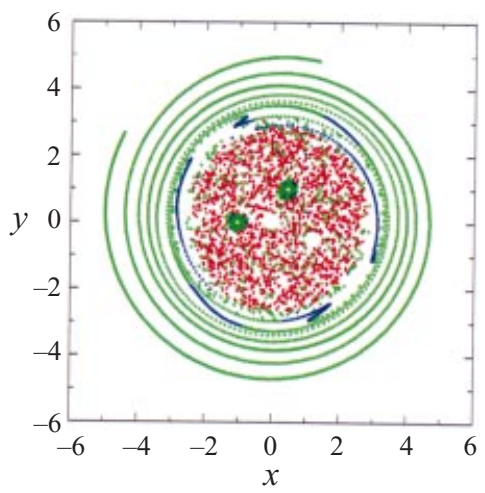

(b)

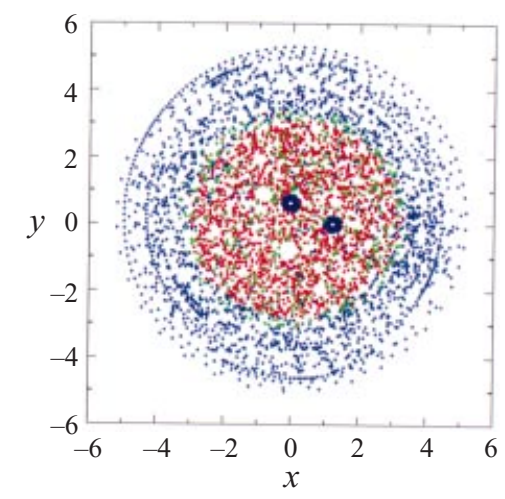

FIGURE 13. As figure 12 but for $\epsilon_{A}=0.5$.

\subsection{Clouds of tracer and the corresponding distribution of Lyapunov exponents}

For our first application of the FTLE analysis we considered an initially collinear distribution of 10000 particles $\left(-5<x_{o}<+5, y_{o}=0.01\right)$. We recorded the position of the particles after some time - we have chosen the times $T_{1}=500$ and $T_{2}=4000$ - and computed the corresponding FTLE distribution. Particles are colour-coded according to the value of the corresponding FTLE. We divided the FTLE into three ranges of values and assigned the colour blue for low values $\left(\lambda\left(\boldsymbol{r}_{o}, T\right)<0.004\right)$, green for intermediate values $\left(0.004<\lambda\left(\boldsymbol{r}_{o}, T\right)<0.02\right)$, and red for high values $\left(\lambda\left(\boldsymbol{r}_{o}, T\right)>0.02\right)$. From figures 12 and 13 it is evident that, for both quasi-periodic and chaotic vortex dynamics, there exist regular islands encircling the vortices. One can, in fact, observe a sharp transition in the FTLE plots as the position along the $x$ axis is varied in the neighbourhood of a vortex, and one can also verify the persistence of these islands (over the range of times under consideration, $T=500-4000$ ).

In the case of quasi-periodic vortex dynamics, there are additional macroscopic islands in the central region (as already observed in $\S 3$ ) and a well-defined boundary between the inner and outer regions. Notice also that in the outer region the FTLE displays a well-defined convergence to zero, consistently with the predominance of regular trajectories for the tracers.

In the case of chaotic vortex motion the scenario is quite different. First we do not observe additional macroscopic islands other than the ones surrounding the vortices 

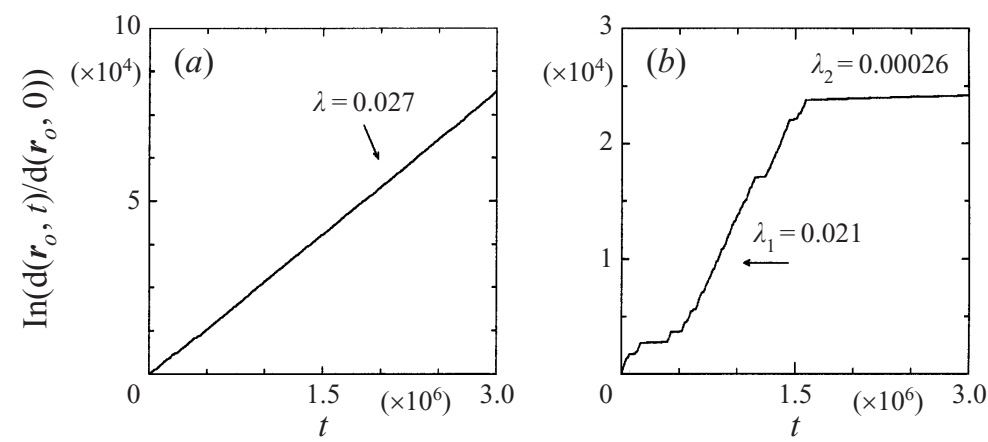

FIGURE 14. Graph of $\ln \left(\mathrm{d}\left(\boldsymbol{r}_{o}, t\right) / \mathrm{d}\left(\boldsymbol{r}_{o}, 0\right)\right)$ for a tracer initially in the inner region (i.e. $\left.r(0)<R_{v}\right)$.

(a) Quasi-periodic vortex dynamics $\left(\epsilon_{S}=0.1\right)$. (b) Chaotic vortex dynamics $\left(\epsilon_{A}=0.5\right)$.

in the central region. Second, as time increases, there is no convergence to lower values of $\lambda_{T}$ in the outer region; on the contrary, there is a slow increase. This is consistent with the existence of outer annular orbits, previously observed in $\S 3$, where the particle can remain trapped for relatively long times, with an essential circular motion and low values of $\lambda_{T}$, before jumping to a new annular orbit or into the central chaotic region, with a consequent increase in the value of $\lambda_{T}$.

\subsection{Time series of stretching: plateaux versus rings}

We have found it useful to study the time series of the stretching rates. We have examined, for both regular and chaotic vortex motion, the dynamics of a particle initially in the central chaotic region (radius less than $R_{v}=\sqrt{L / k}$ ), and followed its evolution and that of a linearized vector, as described by the equations (4.6)-(4.5).

In figure 14 the graphs of the function $\ln \left(\mathrm{d}\left(\boldsymbol{r}_{o}, t\right) / \mathrm{d}\left(\boldsymbol{r}_{o}, 0\right)\right)$ are shown, where $\boldsymbol{r}_{o}$ is the initial location of the particle. The difference between chaotic and quasi-periodic vortex dynamics is manifest. For the quasi-periodic vortex motion we have a welldefined slope, whose corresponding $\lambda$ suggests Lagrangian chaos. For chaotic vortex dynamics there are plateaux alternating with steep slopes. These plateaux and steep portions of the graph (and the corresponding FTLEs) suggest the existence of two separate phases for the tracer dynamics: a regular and a chaotic phase, during which the particle respectively experiences low $\left(\lambda_{T}<0.004\right)$ and high $\left(\lambda_{T}>0.02\right)$ stretching rates.

To understand this process better, we have recorded tracer positions during the plateaux phases, as shown in figure 15. We have found that regions of low stretching rates (low $\lambda_{T}$ ) correspond to trapping of the particle in the outer annular orbits, while regions of high stretching rates (high $\lambda_{T}$ ) correspond to motion in the central chaotic disk. Figure 15 describes then a particle motion with intermittent behaviour, as already seen in $\S 3$ (figure 7), where ordered motion (outer-ring trapping) alternates with chaotic motion (inner-disk trapping).

\subsection{Probability distribution function of finite-time Lyapunov exponents}

The probability distribution function of finite-time Lyapunov exponents (PDFL) has been the object of several studies and many interpretations have been given concerning its shape and the relationship to the structure of the space (Ott 1993; Horita et al. 1990; Chertkov et al. 1995). We have computed the probability distribution function for the FTLE $\lambda(\boldsymbol{r}, T)$ with respect to points along a trajectory, $\boldsymbol{r}(t)$, originating in the central chaotic region, and for different finite times $T$ (with $500<T<20000$ ). 


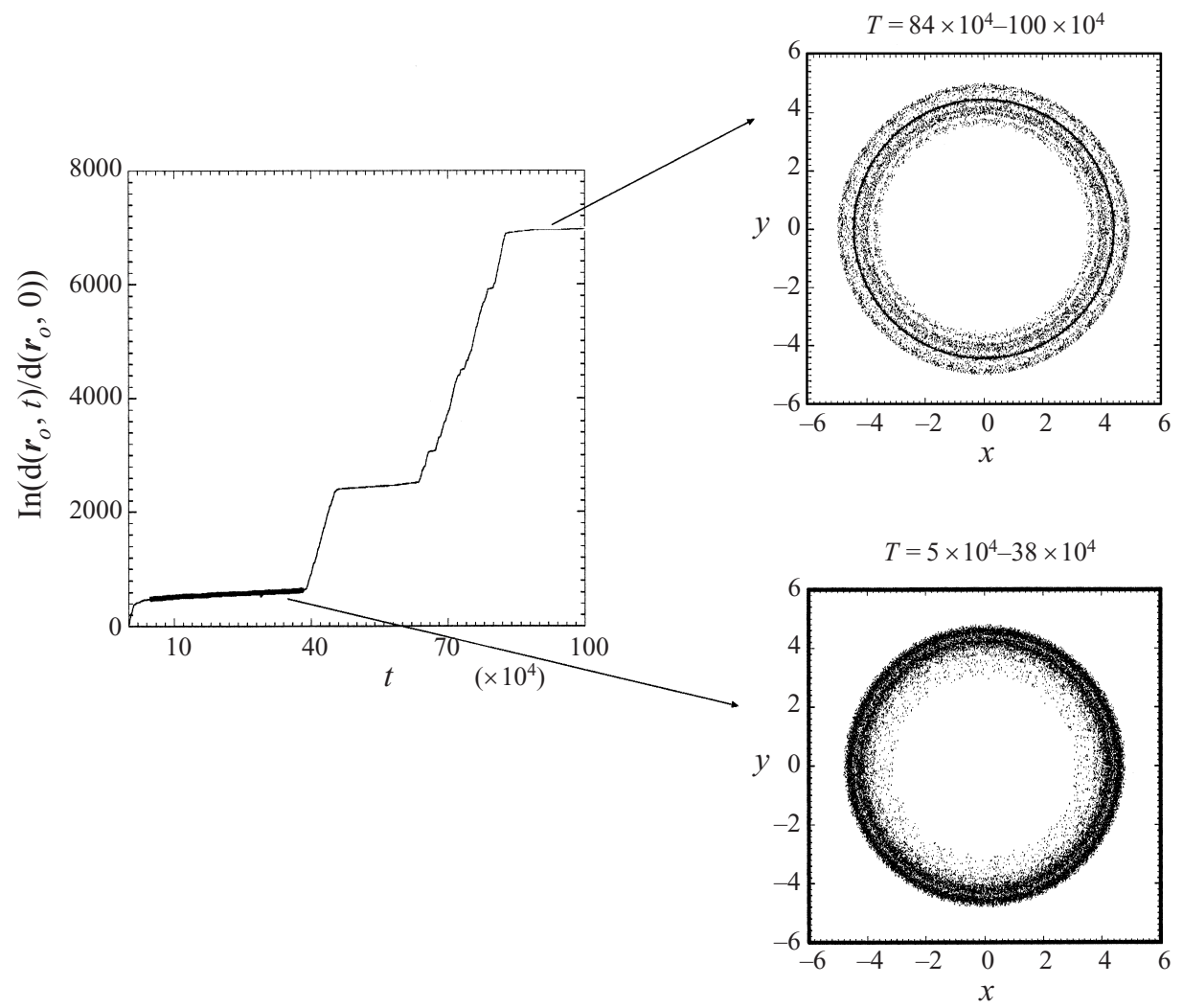

FiguRE 15. The plateau regions in the graph of $\ln \left(\mathrm{d}\left(\boldsymbol{r}_{o}, t\right) / \mathrm{d}\left(\boldsymbol{r}_{o}, 0\right)\right)$ correspond to the chaotic orbit being trapped in rings in the outer region (chaotic vortex dynamics, $\epsilon_{A}=0.5$ ). We have shown as thick lines two different plateaux and the corresponding portions of the orbit.

The FTLE $(\lambda(\boldsymbol{r}, T))$ at a given point $\boldsymbol{r}(t)$ along the trajectory is the local slope of the graph of $\ln (\mathrm{d}(\boldsymbol{r}, t+T) / \mathrm{d}(\boldsymbol{r}, t))$ versus $T$. Comparing the PDFLs for quasi-periodic and chaotic vortex dynamics, dissimilarities are quite noticeable (see figure 16). In the case of quasi-periodic vortex dynamics for short times the PDFLs are unimodal showing a single, quite broad peak, connected with consistently chaotic motion. For longer times a secondary peak centred at $\lambda=0$ appears from islands. As $T$ increases, the peaks separate and become narrower, the primary peak becomes more pronounced and the secondary peak almost disappears. Asymptotically the PDFL exhibits one dominant peak, quite narrow, and non-Gaussian (see figure 17). The non-Gaussian character of the peak agrees with the presence of regular islands inside the chaotic regions. In fact, it has been shown (Shraiman \& Siggia 1994) that the distribution of FTLEs converges to different asymptotic forms depending on whether the system has regular islands or not. Systems without islands display a PDFL that is essentially Gaussian. On the other hand, if regular islands are present, the PDFL shows an asymmetry: the region on the left of the peak shows a longer tail, which is related to the stickiness of tracer trajectories in the neighbourhood of regular islands. In fact, as already pointed out by Horita et al. (1990), there are two distinct regions in the stochastic sea: one is a boundary region encircling regular islands and the other is the chaotic sea itself. Chaotic orbits can wander close to the bounding KAM tori, which are present at the boundary of a regular island, and when they do so, they can become stuck there for a long time due to the presence of semi-permeable barriers, the cantori. 
(a)

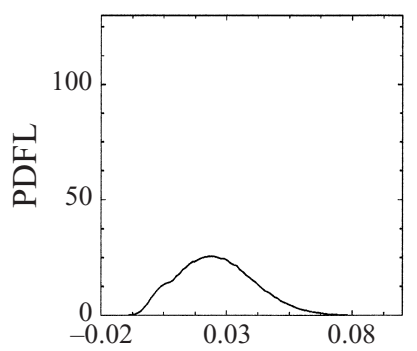

$T=500$

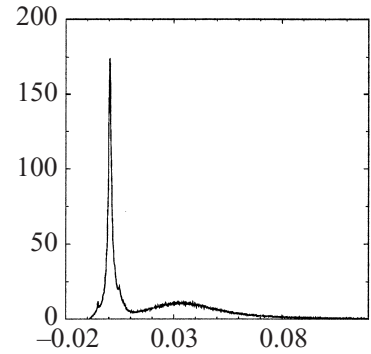

$T=4000$
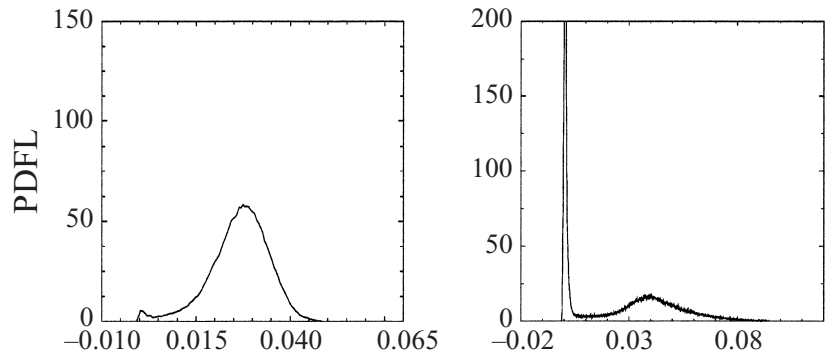

$T=2 \times 10^{4}$
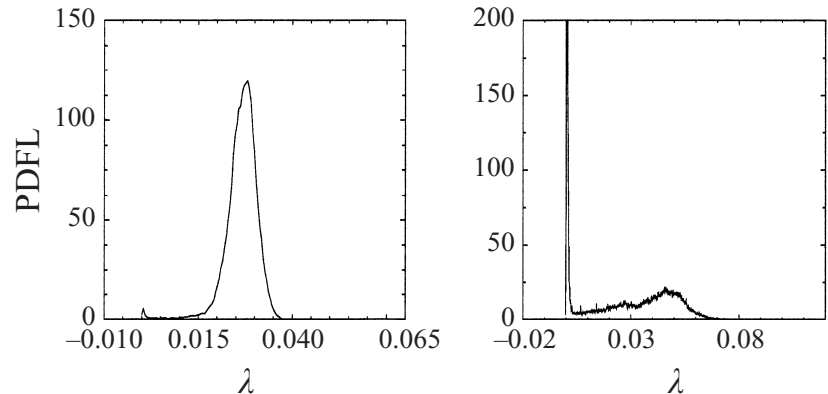

Figure 16. PDFL. (a) Quasi-periodic vortex dynamics $\left(\epsilon_{s}=0.1\right)$ : for short times, $T$, the PDFL shows only one broad chaotic peak $\left(\lambda_{\text {peak }}>0\right)$, whereas for longer times a secondary peak at low $\lambda$ appears, and the main peak becomes more pronounced and narrow. $(b)$ Chaotic vortex dynamics $\left(\epsilon_{A}=0.5\right)$ : the PDFLs show two peaks. The areas under the peaks are of comparable size, suggesting that the particle experiences regular and chaotic dynamics.
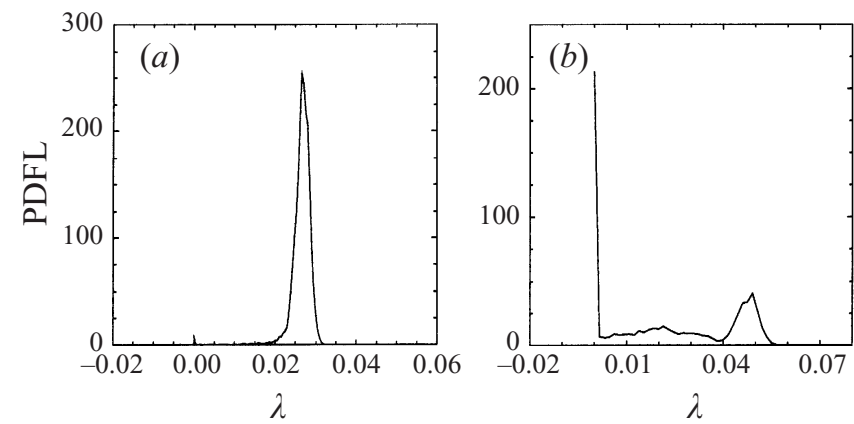

FIGURE 17. PDFL for the finite time $T=100000$. (a) For quasi-periodic vortex dynamics the PDFL asymptotically shows a dominant non-Gaussian peak. (b) For chaotic vortex dynamics the PDFL shows the appearance of an additional peak as time increases. 

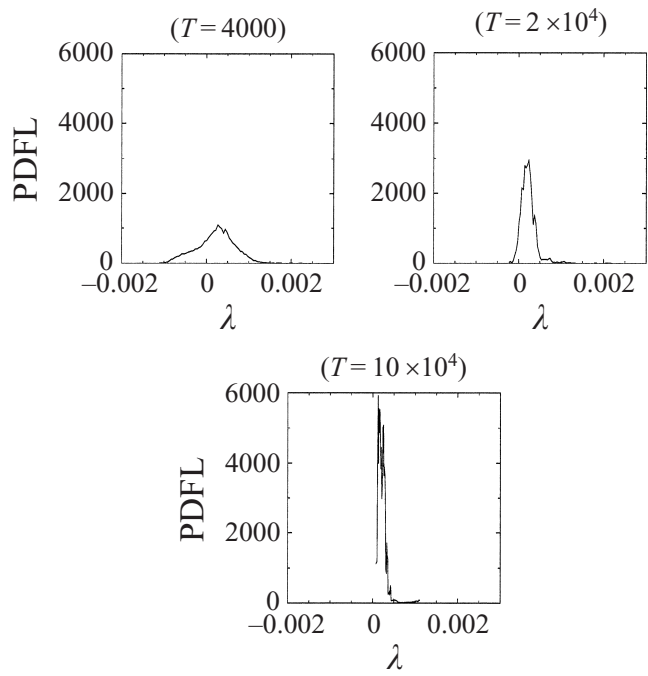

FiguRE 18. PDFL for chaotic vortex dynamics $\left(\epsilon_{A}=0.5\right)$ and for $16 \times 10^{5}<t<48 \times 10^{5}$. During this period of time the tracer is in the outer region (ring-region), as shown in figure 15 . Notice that the PDFL shows one peak which, as time increases, becomes narrower and more pronounced.

Since the Lyapunov exponents on the tori are $\lambda_{1}=\lambda_{2}=0$, orbits that spend a very long time near the tori wind up having abnormally small values of $\lambda(\boldsymbol{r}, T)$. This produces a long tail in the low $\lambda$ values for the corresponding PDFL. Because an orbit has a low probability of crossing a cantorus to enter these regions of phase space, the effect is mostly to raise the tail of the distribution and not to shift the centre. The distribution decays much more slowly than Gaussian on that side. On the other hand, there is no analogous mechanism for producing anomalously large values of $\lambda$ and the distribution is effectively Gaussian on the right (Ott 1993).

The scenario is quite different in the case of chaotic vortex dynamics. The PDFLs exhibit two peaks for short and long times $T$. The persistence of these peaks, even for long $T$, supports the fact that there are two distinct phases for the particle dynamics, which also correspond to two different regions of the phase plane, as we have already noticed in the previous section (see figure 15). The peak at $\lambda=0$ corresponds to the particle dynamics in the outer region: the trajectory is trapped in rings and the particle experiences predominantly regular motion. The peak at $\lambda>0$, on the other hand, corresponds to the dynamics in the central chaotic region. For a very long time, $\uparrow T=100000$, (see figure 17) the PDFL shows an additional peak. This lack of convergence and fragmentation of the PDFL into distinct peaks is again symptomatic of the alternation of different kinds of dynamics. To illustrate this point further, we have analysed the stretching statistics for a ring phase separately. We have chosen the time interval $16 \times 10^{5}<t<48 \times 10^{5}$ which corresponds to a long ring phase, similar to the ones of figure 15 , for a tracer initially in the central chaotic region. As one observes in figure 18, the corresponding PDFL exhibits only one peak, and, as time increases, the peak becomes more pronounced and narrow.

$\dagger$ When using the terminology long and short finite time we mean a time longer or comparable to the dominant frequency of the corresponding vortex spectrum. As, in fact, shown in figure 23 even when the motion of the vortices is chaotic there is a dominant frequency $\omega$ in the vortex spectrum, and a corresponding period $T_{\omega}$ (the turnover time the vortex system). 


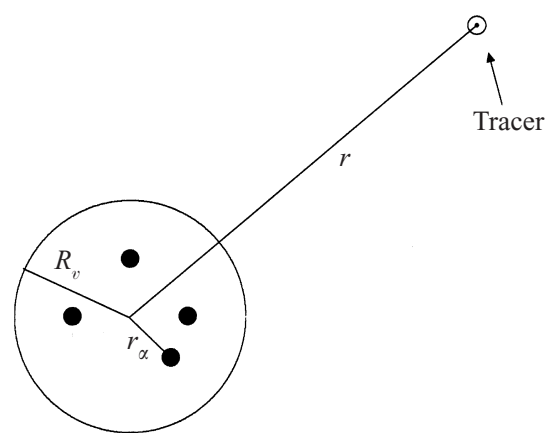

FIGURE 19. Particle in the far-field region: $r_{\alpha}<R_{v} \ll r$, where $r_{\alpha}$ and $r$ are respectively the radial distances to one of the vortices and to the particle, from the origin. The position of a vortex is indicated by

To summarize, stretching analysis confirms the existence of a very well-defined barrier between inner and outer regions in the case of quasi-periodic vortex dynamics. The inner region is chaotic with regular islands embedded in it, whereas the outer region is filled predominantly with regular trajectories. For chaotic vortex motion, the in-out barrier becomes semi-permeable, so some particles initially in the inner region can escape it. Some of the tori of the outer region are partially broken, forming semipermeable barriers. As a consequence, the outer phase space acquires an interwoven ring-like structure. After spending some time trapped in a ring-like orbit, a particle can jump to a new ring or to the central chaotic sea.

\section{Multipole expansion}

In the previous Sections we have examined the tracer dynamics by means of numerical techniques that, even if very useful for short or intermediate times, have some limitations when the long-time behaviour is considered. The long-time behaviour is of particular interest when addressing questions such as: Can a particle escape to infinity? Are there any barriers to transport for the tracer? Is the barrier impermeable barrier or does it allow leaking in and out of particles? How does the nature of the underlying vortex dynamics - especially whether quasi-periodic or chaotic-influence the global structure of the phase space of the tracer? Our numerical studies have already hinted at the possibility of particle escape for the case of chaotic vortex dynamics. We would like then to confirm it analytically.

As noted in $\S 2$ the dynamics of a system of identical vortices is confined to a finite disk of radius $R_{v}=\sqrt{L / k}$. Thus in a regime where the particle is far away from the vortices, with an initial radius $R_{o} \gg R_{v}$ (see figure 19), we can compute a multipole expansion for the tracer velocity field, in the same spirit as far-field expansions in classical electrodynamics. The perturbation parameter for this approximation is $\epsilon=R_{v} / R_{o}$. As a result of the multipole expansion, the equations of evolution for the tracer take the form

$$
\left.\begin{array}{ll}
\dot{r^{2}}=F_{1}\left(r, \theta, r_{\alpha}(t), \theta_{\alpha}(t), \epsilon\right), & \alpha=1, \ldots, 4, \\
\dot{\theta}=\Omega\left(r^{2}\right)+F_{2}\left(r, \theta, r_{\alpha}(t), \theta_{\alpha}(t), \epsilon\right), & \Omega\left(r^{2}\right)=\frac{\sum_{\alpha=1}^{4} k_{\alpha}}{2 \pi r^{2}},
\end{array}\right\}
$$


(b)

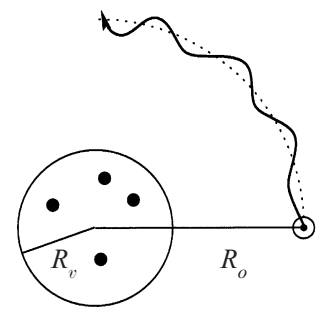

(a)

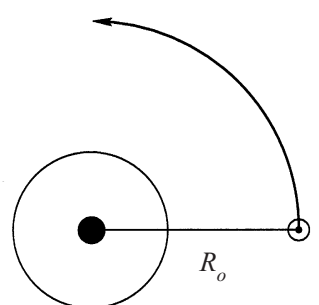

FIGURE 20. Consider a particle initially in the far field, i.e. $R_{v} \ll R_{o}$ (where $r(0)=R_{o}$ ). (a) The main contribution to the velocity field of the particle is the monopole term, i.e. in the zeroth order of the expansion (5.1), the particle dynamics is similar to that in the velocity field of one point vortex (with $K=4 k$ ). (b) The particular vortex dynamics appears only in the higher-order terms of (5.1). The position of a vortex is indicated by

where $(r, \theta)$ are the polar coordinates of the tracer, $\left(r_{\alpha}, \theta_{\alpha}\right)$ are the vortex polar coordinates, and $F_{1}(r, \theta, t, 0)=F_{2}(r, \theta, t, 0)=0$. For a tracer in the velocity field of a single vortex, the equations of motion in action-angle variables are simply

$$
\begin{gathered}
\dot{r}^{2}=0, \\
\dot{\theta}=\Omega\left(r^{2}\right),
\end{gathered}
$$

where $\Omega\left(r^{2}\right)=K /\left(2 \pi r^{2}\right)$. Consequently, the tracer velocity field, (5.1), can be viewed as the sum of a zero-order term (or monopole term), analogous to a one-point-vortex velocity field with vortex strength $K=\sum_{\alpha=1}^{4} k_{\alpha}$ (see figure 20), to which are added corrections of higher order in the expansion parameter $\epsilon$. These depend explicitly on the vortex variables and on their dynamics, i.e.

$$
\begin{aligned}
& F_{1}\left(r, \theta, X_{\alpha}(t), Y_{\alpha}(t), \epsilon\right)=F_{1}(r, \theta, t, \epsilon), \\
& F_{2}\left(r, \theta, X_{\alpha}(t), Y_{\alpha}(t), \epsilon\right)=F_{2}(r, \theta, t, \epsilon) .
\end{aligned}
$$

Our interest is to study how the time dependence of those higher-order terms whether chaotic or quasi-periodic - affects the global transport properties of the tracer dynamics.

In the following paragraphs we will make use of complex variables to simplify the calculation of the far-field expansion, and we will make use of polar coordinates to write the evolution equations explicitly in action-angle variables. We will then consider a truncated version of the far-field equations and discuss a general procedure to find an averaged solution. Finally, we will consider a model in which the coefficients of the truncated far-field equations are replaced by simple functions of time. We will see how diffusive or non-diffusive behaviour of the tracer is related to the choice of the time-dependent functions and, in particular, how random functions can generate diffusion of the tracer. This is done as a first step to investigate global changes in the tracer phase space.

\subsection{Action-angle evolution equations}

In (2.4) the tracer dynamics is written as a set of Hamiltonian equations in canonical coordinates $(x, y)$ using the stream function defined by (2.3) as a Hamiltonian. Making use of the complex variables, $z=x+\mathrm{i} y$ and $z_{\alpha}=X_{\alpha}+\mathrm{i} Y_{\alpha}$, we can introduce a complex 
potential $\Psi$, with derivative $\Psi^{\prime}=\mathrm{d} \Psi / \mathrm{d} z$ as follows:

$$
\Psi(z)=-\frac{1}{2 \pi} \sum_{\alpha=1}^{4} k_{\alpha} \ln \left|z-z_{\alpha}\right|, \quad \Psi^{\prime}(z)=\frac{1}{2 \pi} \sum_{\alpha=1}^{4} k_{\alpha} \frac{1}{z-z_{\alpha}},
$$

so that the tracer equations of motion can be rewritten as

$$
\dot{x}=\operatorname{Im}\left(\Psi^{\prime}(z)\right), \quad \dot{y}=\operatorname{Re}\left(\Psi^{\prime}(z)\right) .
$$

The function $\Psi^{\prime}(z)$ is particularly useful for far-field calculations. In fact, for all $z$ lying outside the disk of radius $R_{v}$ that contains the vortices, we can easily compute the far-field expansion for $\Psi^{\prime}$ :

$$
\Psi^{\prime}(z)=\frac{1}{2 \pi z} \sum_{\alpha=1}^{4} k_{\alpha}+\frac{1}{2 \pi z} \sum_{n=1}^{\infty} \sum_{\alpha=1}^{4} k_{\alpha}\left(\frac{z_{\alpha}}{z}\right)^{n},
$$

where $|z|>R_{v}$. Expressing $z$ and $z_{\alpha}$ in terms of polar coordinates,

$$
z=r \mathrm{e}^{\mathrm{i} \theta} \quad \text { and } \quad z_{\alpha}=r_{\alpha} \mathrm{e}^{\mathrm{i} \theta_{\alpha}},
$$

$\Psi^{\prime}$ can be written as a function of $(r, \theta)$ as follows:

$$
\Psi^{\prime}=\frac{\mathrm{e}^{-\mathrm{i} \theta}}{2 \pi r} \sum_{\alpha=1}^{4} k_{\alpha}+\frac{1}{2 \pi r} \sum_{n=1}^{\infty} \sum_{\alpha=1}^{4} k_{\alpha}\left(\frac{r_{\alpha}}{r}\right)^{n} \mathrm{e}^{\mathrm{i}\left[n\left(\theta_{\alpha}-\theta\right)-\theta\right]} .
$$

The tracer evolution equations then become

$$
\begin{gathered}
\dot{r}^{2}=\frac{1}{\pi} \sum_{n=1}^{\infty} \sum_{\alpha=1}^{4} k_{\alpha}\left(\frac{r_{\alpha}}{r}\right)^{n} \sin \left[n\left(\theta_{\alpha}-\theta\right)\right], \\
\dot{\theta}=\frac{1}{2 \pi r^{2}} \sum_{\alpha=1}^{4} k_{\alpha}+\frac{1}{2 \pi r^{2}} \sum_{n=1}^{\infty} \sum_{\alpha=1}^{4} k_{\alpha}\left(\frac{r_{\alpha}}{r}\right)^{n} \cos \left[n\left(\theta_{\alpha}-\theta\right)\right] .
\end{gathered}
$$

Since the vortex motion is confined $\left(r_{\alpha}<R_{v}\right)$, as we move farther from the vortex region, i.e. in the limit $r \rightarrow \infty$, (5.8) take the form of (5.2). We recover the equations for a particle in the field of a single point vortex whose circulation is $K=\sum_{\alpha=1}^{4} k_{\alpha}$. The coordinates $\left(r^{2}, \theta\right)$ are then the action-angle variables for this system and the particle trajectories are circular orbits, with constant action $r^{2}=R_{o}^{2}$ and corresponding angular frequency $\Omega\left(R_{o}^{2}\right)=K /\left(2 \pi R_{o}^{2}\right)$.

When higher-order terms in the perturbation expansion are included in the equations of motion, these circular trajectories are perturbed, and tracer diffusion may occur (see figure 20).

To better view this expansion, we make use of the dimensionless variables $\rho$ and $\rho_{\alpha}$, as defined by

$$
\rho=\frac{r}{R_{o}}, \quad \rho_{\alpha}=\frac{r_{\alpha}}{R_{v}} .
$$

We then rewrite (5.8) in the form given in (5.1) with

$$
\left.\begin{array}{l}
F_{1}\left(\rho, \theta, \rho_{\alpha}(t), \theta_{\alpha}(t), \epsilon\right)=\frac{1}{\pi R_{o}^{2}} \sum_{n=1}^{\infty} \epsilon^{n}\left(\sum_{\alpha=1}^{4} k_{\alpha}\left(\frac{\rho_{\alpha}}{\rho}\right)^{n} \sin \left[n\left(\theta_{\alpha}-\theta\right)\right]\right), \\
F_{2}\left(\rho, \theta, \rho_{\alpha}(t), \theta_{\alpha}(t), \epsilon\right)=\frac{1}{2 \pi R_{o}^{2} \rho^{2}} \sum_{n=1}^{\infty} \epsilon^{n}\left(\sum_{\alpha=1}^{4} k_{\alpha}\left(\frac{\rho_{\alpha}}{\rho}\right)^{n} \cos \left[n\left(\theta_{\alpha}-\theta\right)\right]\right),
\end{array}\right\}
$$

where $\epsilon=R_{v} / R_{o}$ and $r=\rho R_{o}$. 
Notice that the motion of the particle in the far field is essentially governed by two times: a fast time, $T_{v}$, and a slow time, $T_{T}=\left(4 \pi^{2} R_{o}^{2}\right) / K \sim \epsilon^{-2} T_{v}$, which are related, respectively, to the vortex motion and to the zero-order circular motion of the tracer. Equations (5.1) are then in a form to which averaging methods can be applied. Approximate solutions $\rho(t)$ can be computed and an estimate for a possible diffusion coefficient $D$ can be found. Here $D$ is defined by $\left\langle(r-\langle r\rangle)^{2}\right\rangle=R_{o}^{2}\left\langle(\rho-\langle\rho\rangle)^{2}\right\rangle \sim D t$.

\subsection{A simple stochastic model}

Our goal in this Section will be to relate the vortex dynamics to possible diffusive behaviour of the tracer. We are interested in determining the solution $\boldsymbol{r}(t)$ of (5.1) and in computing the corresponding mean-squared distance $\left\langle[\Delta r(t)]^{2}\right\rangle \equiv\left\langle r^{2}\right\rangle-\langle r\rangle^{2}$. For this purpose we rewrite the radial-angle equations, (5.1), in terms of the rescaled vortex momenta, $P_{x}$ and $P_{y}$, and quadrupole moment components, $Q_{1}$ and $Q_{2}$, giving, in the rescaled variables

$$
\begin{aligned}
\frac{\mathrm{d} \rho^{2}}{\mathrm{~d} t}= & \frac{1}{\pi R_{o}^{2}}\left\{\frac{\epsilon}{\rho}\left(P_{y} \cos \theta-P_{x} \sin \theta\right)+\frac{\epsilon^{2}}{\rho^{2}}\left(Q_{1} \cos (2 \theta)-Q_{2} \sin (2 \theta)\right)+O\left(\epsilon^{3}\right)\right\} \\
\frac{\mathrm{d} \theta}{\mathrm{d} t}= & \frac{1}{2 \pi R_{o}^{2} \rho^{2}}\left\{\sum_{\alpha=1}^{4} k_{\alpha}+\frac{\epsilon}{\rho}\left(P_{x} \cos \theta+P_{y} \sin \theta\right)\right. \\
& \left.+\frac{\epsilon^{2}}{\rho^{2}}\left(Q_{2} \cos (2 \theta)+Q_{1} \sin (2 \theta)\right)+O\left(\epsilon^{3}\right)\right\}
\end{aligned}
$$

where

$$
\left.\begin{array}{rl}
P_{x} & =\sum_{\alpha=1}^{4} k_{\alpha} \rho_{\alpha} \cos \theta_{\alpha}=\frac{1}{R_{o}} \sum_{\alpha=1}^{4} k_{\alpha} X_{\alpha}, \\
P_{y} & =\sum_{\alpha=1}^{4} k_{\alpha} \rho_{\alpha} \sin \theta_{\alpha}=\frac{1}{R_{o}} \sum_{\alpha=1}^{4} k_{\alpha} Y_{\alpha}, \\
Q_{1}(t) & =\sum_{\alpha=1}^{4} k_{\alpha} \rho_{\alpha}^{2} \sin \left(2 \theta_{\alpha}\right)=\frac{1}{R_{o}^{2}} \sum_{\alpha=1}^{4} k_{\alpha} 2 X_{\alpha} Y_{\alpha}, \\
Q_{2}(t) & =\sum_{\alpha=1}^{4} k_{\alpha} \rho_{\alpha}^{2} \cos \left(2 \theta_{\alpha}\right)=\frac{1}{R_{o}^{2}} \sum_{\alpha=1}^{4} k_{\alpha}\left(X_{\alpha}^{2}-Y_{\alpha}^{2}\right) .
\end{array}\right\}
$$

As previously seen in $\S 2, P_{x}$ and $P_{y}$ are constants of motion. It is possible to choose a reference frame in which $P_{x}=P_{y}=0$, and then the first perturbative corrections in (5.1) are second order in $\epsilon$.

For a given dynamics of the vortices, the solutions of equations (5.11) can be expanded in the perturbation parameter $\epsilon$ to give

$$
\rho=\sum_{n=0}^{\infty} \epsilon^{n} \rho_{n}=\rho_{o}+\epsilon^{2} \rho_{2}+O\left(\epsilon^{3}\right), \quad \theta=\sum_{n=0}^{\infty} \epsilon^{n} \theta_{n}=\theta_{0}+\epsilon^{2} \theta_{2}+O\left(\epsilon^{3}\right),
$$

and it can be easily shown that

$$
\rho_{0}(t)=1 \quad \theta_{o}(t)=\Omega_{o} t+\theta_{o}(0), \quad \Omega_{o}=\frac{1}{2 \pi R_{o}^{2}} \sum_{\alpha=1}^{4} k_{\alpha},
$$


(a)
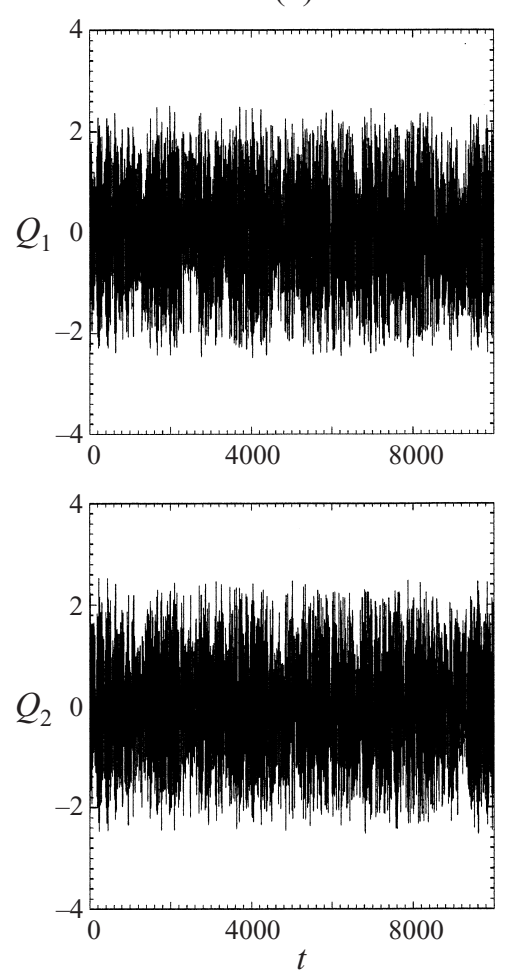

(b)
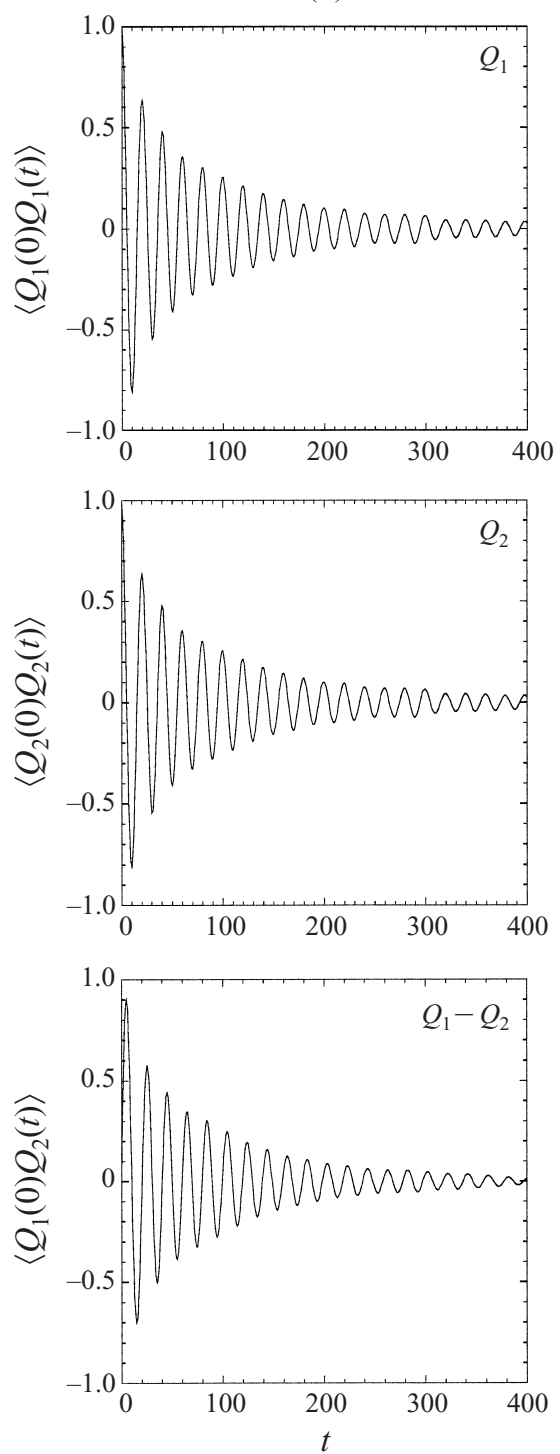

Figure 21. $Q_{1}$ and $Q_{2}$ statistics for a case of chaotic vortex dynamics $\left(\epsilon_{A}=0.5\right)$. (a) Times series for $Q_{1}(t)=k \sum_{\alpha=1}^{4} 2 X_{\alpha}(t) Y_{\alpha}(t)$ and $Q_{2}(t)=k \sum_{\alpha=1}^{4}\left(X_{\alpha}^{2}(t)-Y_{\alpha}^{2}(t)\right)$. (b) Correlation and autocorrelation functions numerically calculated from the time series of $Q_{1}(t)$ and $Q_{2}(t)$.

where $\theta_{o}(0)$ is the initial phase of the particle. There is no loss of generality if we set $\theta_{o}(0)=0$. The second-order terms, $\rho_{2}$ and $\theta_{2}$, are the first non-zero perturbative terms, and, consequently, the mean-squared distance will be, to leading order,

$$
\left\langle[\Delta \rho(t)]^{2}\right\rangle=\epsilon^{4}\left(\left\langle\rho_{2}^{2}\right\rangle-\left\langle\rho_{2}\right\rangle^{2}\right)+O\left(\epsilon^{5}\right) .
$$


To compute $\left\langle\rho_{2}^{2}\right\rangle$ and $\left\langle\rho_{2}\right\rangle^{2}$ we have to solve the corresponding evolution equations

$$
\left.\begin{array}{l}
\frac{\mathrm{d} \rho_{2}}{\mathrm{~d} t}=\frac{1}{2 \pi R_{o}^{2}}\left\{Q_{1}(t) \cos \left(2 \Omega_{o} t\right)-Q_{2}(t) \sin \left(2 \Omega_{o} t\right)\right\}, \\
\frac{\mathrm{d} \theta_{2}}{\mathrm{~d} t}=\frac{1}{2 \pi R_{o}^{2}}\left\{Q_{2}(t) \cos \left(2 \Omega_{o} t\right)+Q_{1}(t) \sin \left(2 \Omega_{o} t\right)\right\},
\end{array}\right\}
$$

whose radial solution can be written formally as

$$
\rho_{2}(t)=\frac{1}{2 \pi R_{o}^{2}} \int_{0}^{t}\left\{Q_{1}\left(t^{\prime}\right) \cos \left(2 \Omega_{o} t^{\prime}\right)-Q_{2}\left(t^{\prime}\right) \sin \left(2 \Omega_{o} t^{\prime}\right)\right\} \mathrm{d} t^{\prime} .
$$

Notice that $\rho_{2}(t)$ depends on the vortex dynamics through the coefficients $Q_{1}(t)$ and $Q_{2}(t)$ defined in (5.12). In the case of chaotic vortex dynamics, we do not have an analytical expression for $Q_{1}(t)$ and $Q_{2}(t)$ nor, consequently, for $\rho_{2}(t)$. Instead, we can obtain numerical solutions and compute the corresponding time series for the coefficients $Q_{1}(t)$ and $Q_{2}(t)$ (see figure 21a). For a specific asymmetric vortex configuration $\left(\epsilon_{A}=0.5\right)$, we have analysed the statistical behaviour of these time series and found that the process is stationary, i.e. the statistical quantities of interest are time-translation invariant. For example, $\left\langle Q_{1}(0) Q_{2}(\tau)\right\rangle=\left\langle Q_{1}(t) Q_{2}(t+\tau)\right\rangle$. Stationarity is an important property, since it allows us to have a well-defined notion of correlations, autocorrelations, and average functions. We find that the numerical correlations are well fitted by the following model:

$$
\left.\begin{array}{rl}
\left\langle Q_{1}(0) Q_{1}(\tau)\right\rangle & =A_{11} \mathrm{e}^{-\lambda \tau} \cos (\omega \tau), \\
\left\langle Q_{2}(0) Q_{2}(\tau)\right\rangle & =A_{22} \mathrm{e}^{-\lambda \tau} \cos (\omega \tau), \\
\left\langle Q_{1}(0) Q_{2}(\tau)\right\rangle & =A_{12} \mathrm{e}^{-\lambda \tau} \sin (\omega \tau), \\
\left\langle Q_{1}(\tau)\right\rangle & =0, \quad\left\langle Q_{2}(\tau)\right\rangle=0,
\end{array}\right\}
$$

where $\left\langle Q_{1}(t)\right\rangle \equiv(1 / N) \sum_{k=1}^{N} Q_{1}(t+\Delta t k), \Delta t$ is a constant time step, $N$ is the number of time steps, and $A_{11}, A_{22}$ and $A_{12}$ are constants (see figure $21 b$ ).

In the case of chaotic motion we introduce a model in which $Q_{1}$ and $Q_{2}$ are assumed to be stochastic variables whose statistics are described in equations (5.16). In this case $\langle\cdot\rangle$ denotes ensemble averages over different sets of initial vortex configurations. We can then multiply (5.14) by $\rho_{2}$,

$$
\begin{aligned}
\rho_{2} \frac{\mathrm{d} \rho_{2}}{\mathrm{~d} t}= & \frac{1}{4 \pi^{2} R_{o}^{4}} \int_{0}^{t}\left(Q_{1}\left(t^{\prime}\right) \cos \left(2 \Omega_{o} t^{\prime}\right)-Q_{2}\left(t^{\prime}\right) \sin \left(2 \Omega_{o} t^{\prime}\right)\right) \\
& \times\left(Q_{1}(t) \cos \left(2 \Omega_{o} t\right)-Q_{2}(t) \sin \left(2 \Omega_{o} t\right)\right) \mathrm{d} t^{\prime},
\end{aligned}
$$

and compute the ensemble average,

$$
\begin{aligned}
\frac{1}{2} \frac{\mathrm{d}\left\langle\rho_{2}^{2}\right\rangle}{\mathrm{d} t}= & \frac{1}{4 \pi^{2} R_{o}^{4}} \int_{0}^{t}\left(\left\langle Q_{1}(t-\tau) Q_{1}(t)\right\rangle \cos \left[2 \Omega_{o}(t-\tau)\right] \cos \left(2 \Omega_{o} t\right)\right. \\
& +\left\langle Q_{2}(t-\tau) Q_{2}(t)\right\rangle \sin \left[2 \Omega_{o}(t-\tau)\right] \sin \left(2 \Omega_{o} t\right) \\
& -\left\langle Q_{1}(t-\tau) Q_{2}(t)\right\rangle \cos \left[2 \Omega_{o}(t-\tau)\right] \sin \left(2 \Omega_{o} t\right) \\
& \left.-\left\langle Q_{1}(t) Q_{2}(t-\tau)\right\rangle \cos \left(2 \Omega_{o} t\right) \sin \left[2 \Omega_{o}(t-\tau)\right]\right) \mathrm{d} \tau
\end{aligned}
$$

where we have made use of the change of variables $t^{\prime}=t-\tau$. Substituting (5.16) in 
(5.17) and integrating both sides of (5.17), we find

$$
\left\langle\rho_{2}^{2}\right\rangle(t)=D_{1} t+D_{2}+\mathrm{e}^{-\lambda t} B(t)+C(t),
$$

where $B(t)$ and $C(t)$ are oscillating functions and $D_{1}$ and $D_{2}$ are

$$
\begin{aligned}
& D_{1}=\frac{A_{11}+A_{22}}{8 \pi^{2} R_{o}^{4}}\left\{\frac{\lambda}{\lambda^{2}+\left(\omega+2 \Omega_{o}\right)^{2}}+\frac{\lambda}{\lambda^{2}+\left(\omega-2 \Omega_{o}\right)^{2}}\right\}, \\
& D_{2}=-\frac{A_{11}+A_{22}}{8 \pi^{2} R_{o}^{4}}\left\{\frac{\lambda^{2}}{\left[\lambda^{2}+\left(\omega+2 \Omega_{o}\right)^{2}\right]^{2}}+\frac{\lambda^{2}}{\left[\lambda^{2}+\left(\omega-2 \Omega_{o}\right)^{2}\right]^{2}}\right\} .
\end{aligned}
$$

By analogy, we can estimate $\left\langle\rho_{2}\right\rangle(t)$ by averaging (5.15)

$$
\left\langle\rho_{2}\right\rangle(t)=\frac{1}{2 \pi R_{o}^{2}} \int_{0}^{t}\left\{\left\langle Q_{1}(\tau)\right\rangle \cos \left(2 \Omega_{o} \tau\right)-\left\langle Q_{2}(\tau)\right\rangle \sin \left(2 \Omega_{o} \tau\right)\right\} \mathrm{d} \tau=0 .
$$

For times that are long compared with characteristic time $1 / \lambda$, we then find behaviour of the type

$$
\left\langle[\Delta \rho(t)]^{2}\right\rangle=\epsilon^{4} D_{1} t,
$$

that in the original variables becomes

$$
\left\langle[\Delta r(t)]^{2}\right\rangle=R_{o}^{2}\left\langle[\Delta \rho(t)]^{2}\right\rangle=R_{o}^{2} \epsilon^{4} D_{1} t .
$$

This diffusion-like behaviour has a position-dependent diffusion coefficient, $R_{o}^{2} \epsilon^{4} D_{1}$, decaying quite rapidly with the radial distance $R_{o}$. Diffusion is then expected to fall off as $R_{o} \rightarrow \infty$. In fact, in the limit $R_{o} \rightarrow \infty$,

$$
R_{o}^{2} \epsilon^{4} D_{1} \rightarrow D=\frac{D_{o}}{R_{o}^{6}}
$$

where

$$
D_{o}=R_{v}^{4} \frac{A_{11}-A_{22}}{8 \pi^{2}}\left\{\frac{2 \lambda}{\lambda^{2}+\omega^{2}}\right\} .
$$

Note that, for the case of quasi-periodic vortex motion, the numerical correlations are quite similar to those described by (5.16), setting $\lambda=0$, i.e.

$$
\left.\begin{array}{l}
\left\langle Q_{1}(0) Q_{1}(\tau)\right\rangle=B_{11} \cos \left(\omega_{1} \tau\right), \\
\left\langle Q_{2}(0) Q_{2}(\tau)\right\rangle=B_{22} \cos \left(\omega_{1} \tau\right), \\
\left\langle Q_{1}(0) Q_{2}(\tau)\right\rangle=B_{12} \sin \left(\omega_{1} \tau\right),
\end{array}\right\}
$$

where $B_{11}, B_{12}, B_{22}$ and $\omega_{1}$ are constants. Then by considering the limit $\lambda \rightarrow 0$ in (5.19) we have that $D_{1} \rightarrow 0$ (or equivalently $D \rightarrow 0$ in (5.22)) unless $\omega= \pm 2 \Omega_{o}$, i.e. we have no diffusion for the quasi-periodic case unless we have a resonance between the frequency of the perturbative term, $\omega$, and the frequency of the unperturbed orbit $\Omega_{0}$.

Note as well that, considering the limit $\lambda \rightarrow \infty$ in (5.19), which corresponds to a very short characteristic correlation time or more randomized vortex motion, we have that $D_{1} \rightarrow 0$.

The predictions of our model are in agreement with our numerical simulations of $\S 3$. To illustrate this point, we compute the numerical estimate of $\left\langle\Delta r^{2}\right\rangle$ for the cloud of tracers already considered in $\S 3.2$ and whose temporal evolution is summarized in 

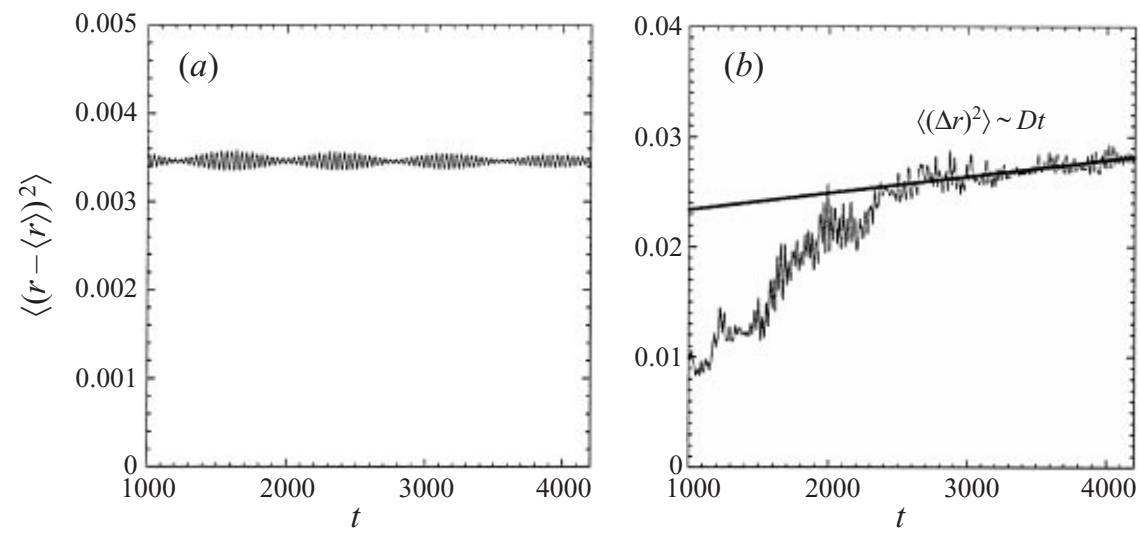

FiguRE 22. Diffusion of a cloud of tracers initially distributed uniformly over a thin annulus centred at $R=4$ in the outer region. In the case of $(a)$ quasi-periodic vortex dynamics $\left(\epsilon_{S}=0.1\right)\left\langle[\Delta r]^{2}\right\rangle(t)$ is bounded and oscillates as a function of time: diffusion does not occur. Meanwhile, in the case of (b) chaotic vortex motion $\left(\epsilon_{A}=0.5\right)\left\langle[\Delta r]^{2}\right\rangle(t) \sim D t$ : diffusion occurs, as also shown in figure 11 .

figure 11; we take $\left\langle\Delta r^{2}\right\rangle$ to be the average over the number of tracers $N T$, i.e.

$$
\left\langle[\Delta r]^{2}\right\rangle(t)=\left\langle[r(t)-\langle r(t)\rangle]^{2}\right\rangle=\frac{1}{N T} \sum_{i=1}^{N T}\left(r_{i}(t)-\langle r(t)\rangle\right)^{2} .
$$

We find that in the case of chaotic vortex motion, after some transient initial time, $\left\langle[\Delta r]^{2}\right\rangle$ shows a linear increase with time (see figure $22 a$ ), as predicted by our model (5.21), confirming the diffusive behaviour of tracers for this vortex dynamics.

Furthermore for the case of quasi-periodic vortex motion, $\left\langle\Delta r^{2}\right\rangle$ exhibits an oscillating behaviour of quite small amplitude (see figure $22 b$ ), confirming the absence of diffusion, as also predicted by our model.

As one last remark about our analytical model, we note that by substituting the expression for $D,(5.22)$, into the corresponding diffusion equation (McQuarrie 1976),

$$
\frac{\partial p}{\partial t}=\frac{1}{r} \frac{\partial}{\partial r}\left(r D \frac{\partial p}{\partial r}\right)
$$

we find

$$
p(r, t)=\frac{p_{o}}{16 D_{o} t} \exp \left(-\frac{r^{8}}{16 D_{o} t}\right),
$$

where $p(r, t)$ is the probability density of finding a particle at a distance $r$ from the origin at a time $t$. This is a sub-diffusive regime: at a given time $t$, the probability of finding a particle falls off as a function of the distance $r$ much more rapidly than in a normal diffusion process. The characteristic distance from the origin at which the probability is reduced by a factor of $1 / \mathrm{e}$ is $r_{c}=\left(16 D_{o} t\right)^{1 / 8}$, whereas for a normal diffusion process it would be $\left(4 D_{o} t\right)^{1 / 2}$.

\section{Rings}

One of the most striking features of the tracer dynamics is that the tracer can become trapped for long times in ring orbits, even at distances that are moderate compared to typical inter-vortex separations. Similar features are observed, for example, in the rings of Saturn and in accretion disks. For our system, the stability of these 

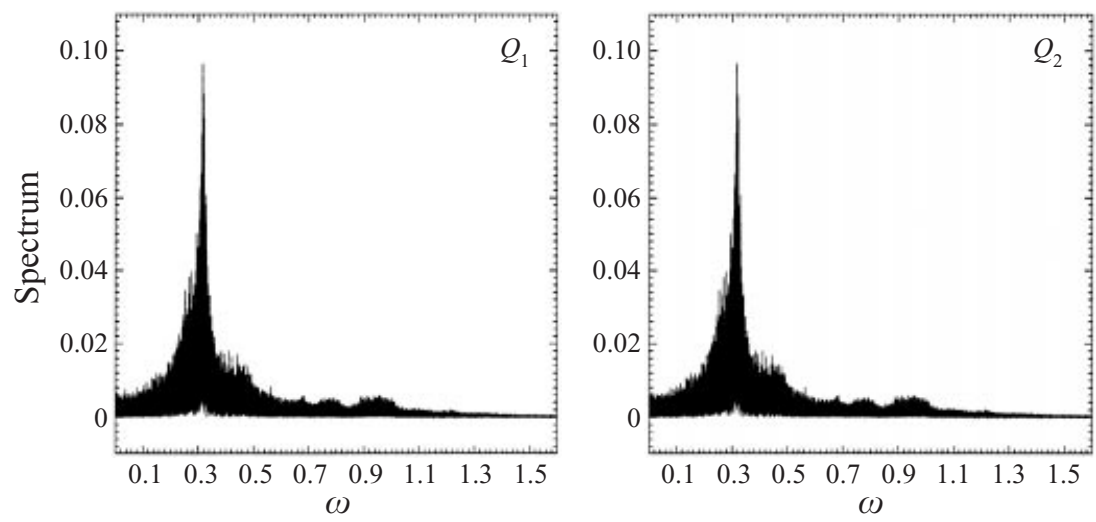

FIGURE 23. Power spectrum for $Q_{1}(t)=k \sum_{\alpha=1}^{4} 2 X_{\alpha} Y_{\alpha}$ and $Q_{2}(t)=k \sum_{\alpha=1}^{4}\left(X_{\alpha}^{2}-Y_{\alpha}^{2}\right)$ for a case of chaotic vortex dynamics $\left(\epsilon_{A}=0.5\right)$.

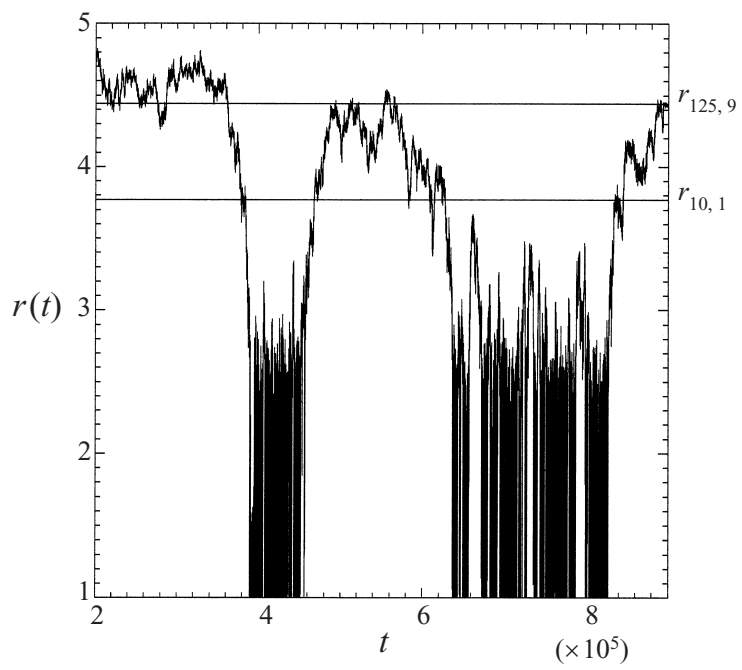

FIgURE 24. Tracer radial distance $r$ as a function of time $t$ for a case of chaotic vortex motion $\left(\epsilon_{A}=0.5\right)$.

outer orbits depends strongly on whether the vortex dynamics is quasi-periodic or chaotic, with completely stable orbits on tori in the former case but with temporary trapping followed by jumps to new radii in the latter. Using the multipole expansion of $\S 5$, for tracers in the far field, the tracer velocity is decomposed into an integrable part plus a time-dependent perturbation, which can be periodic, quasi-periodic or chaotic depending on the corresponding vortex dynamics. The KAM theorem (Arnold 1980) indicates that tori persist in the case of periodic or quasi-periodic time perturbation (Simó 1994) but at the moment an analogous theory for chaotic perturbations does not exist.

We do observe numerically that the tracer remains for long times in ring orbits at approximately fixed radius, rather like KAM tori, but then jumps to new ring orbits at different radii at random times. To understand this behaviour we looked more carefully at the power spectrum of a vortex coordinate: it consists of a broad-band spectrum with a well-pronounced peak. The same is true for the coefficients, $Q_{1}$ 
and $Q_{2}$, which appear in the second-order term of (5.14) (see figure 23). This peak has a natural physical explanation. Since the vortices have the same sign and are few in number, there exists a mean-field turnover time associated with the averaged vortex motion. Therefore the existence of this pronounced peak in the spectrum is explained by the presence of a dominant periodicity in the vortex motion, estimated roughly by $\omega \sim 2 k / \pi \tilde{R}$ ( $\tilde{R}$ is an average vortex radial distance). We conjecture that, under the effect of the perturbation, the most stable tracer orbits are the ones whose corresponding unperturbed frequency $\Omega(r)$ does not resonate with the mean-field frequency $\omega$. As result, a tracer is trapped for a moderately long time in a given ring orbit, until, in its diffusive wanderings, it hits a resonance, i.e. it reaches a radial distance $r$ for which $\Omega$ resonates with $\omega$ as in $m \Omega(r)=n \omega, n, m \in \boldsymbol{Z}$, and then it is likely to be kicked to a new orbit. From our simulations (figure 24) it appears that not all the resonances are active, at least not all at the same time. This is a well-known problem in celestial mechanics and it appears to be related to the 'strength' of each resonance (see Morbidelli \& Guzzo 1996 and references therein) at a given time.

To conclude this Section, we can anticipate that such a distinction between chaotic and quasi-periodic vortex dynamics for a tracer in the far field is going to be relevant up to radial distances $r$ less than a critical distance $R_{c}\left(R_{c} \gg R_{v}\right)$. Following the work of Celletti and coworkers (Celletti \& Falcolini 1988; Celletti \& Chierchia 1987), where the case of four vortices with an initial axial symmetric configuration was considered, one can foresee that for perturbation parameters $\epsilon$ smaller than some critical value $\epsilon<\epsilon_{c}$ (or equivalently $r>R_{c}$ ) the vortex dynamics becomes quasi-integrable (i.e. most of the tori are preserved under the effect of the perturbation). Notice that our definition of a perturbation parameter $\epsilon$ differs from the one given by Celletti and coworkers. Nevertheless the qualitative argument remains the same. Unfortunately we do not yet have an analytical estimate for $R_{c}$ for the general case.

\section{Conclusions}

Both the tracer and the vortex motions are characterized by the existence of two length scales $R_{v}=\sqrt{L / k}$ and $R_{c}$ (with $R_{v} \ll R_{c}$ ), $R_{c}$ being an asymptotic length scale which remains to be estimated analytically. The tracer phase space is found to be partitioned into inner and outer regions. The in-out barrier location has a lower-bound estimate at $r \sim R_{v}$.

There are striking differences in the tracer dynamics between the cases of a driving periodic, quasi-periodic or chaotic vortex motion. Such differences arise predominantly at length scales smaller than $R_{c}$.

In the case of periodic vortex motion, the tracer dynamics is integrable and therefore trajectories in both inner and outer regions are on tori (see figure 4). Mixing and diffusion are inhibited.

In the case of quasi-periodic vortex motion, the in-out boundary represents a well-defined barrier to transport, separating the central chaotic region, punctured by regular islands, from the outer region, where the tracer trajectories are on tori. Mixing takes place only in the central region which exhibits in this case a lobe structure, with several islands in addition to the obvious ones encircling the vortices (figure 8 and figure 12).

On the other hand, in the case of chaotic vortex motion the in-out barrier breaks down. A particle initially in the central region can exit and remain partially trapped in a ring-like orbit for a long time, falling back intermittently into the central chaotic sea (figure 7). As already observed by Babiano et al. (1994), the central region is in 
this case a rather homogeneous disk except for the regular islands which are carried with the individual vortices. For tracers in the outer region, our stochastic model for the velocity field predicts a diffusive behaviour, with a diffusion coefficient $D \sim r^{-1 / 6}$ which decays quite strongly with the radial distance $r$. Diffusion is then expected to fall off quite rapidly.

However, at length scales larger than $R_{c}\left(r>R_{c} \gg R_{v}\right)$, the tracer dynamics appears quite similar (at the mean-field level) for the three classes of vortex motion. In all cases, particles are trapped in a disk moving with the four vortices. The system of vortices can then be considered as a single cluster which acts on a particle in the far field as a single vortex. We can anticipate that clusters of point vortices will give rise to interesting mixing dynamics. Each cluster can be thought of as carrying a mixed cloud. Particles will diffuse out of each vortex region very slowly. In the field of the other clusters, each cluster behaves mainly as a point vortex, as shown in figure $8(e, f)$ for the case of clusters of two vortices. One can extrapolate this line of reasoning and picture clusters of clusters and so on, each individual cluster carrying its own well-mixed cloud. In this context, the existence for a given cluster of an intermediate far-field region with either a stable or an unstable ring-like structure seems to be of importance. In fact, when clusters are sufficiently close, the instability or the stability of their external orbits will respectively allow or inhibit particle exchange between the clusters.

The authors would like to thank L. P. Kadanoff, N. Lebovitz, W. R. Young and V. Rom-Kedar for very stimulating discussions and G. Huber and S. C. Creagh for reading the first draft of this paper. A special thanks to the Laboratoire d'Hydrodynamique de l'Ecole polytechnique (Palaiseau, France) for its hospitality and support during the the last period of revision of this paper. This research was supported by NSF ATM89-20589, NSF-DMR 941560 and CNR (division of mathematical sciences).

\section{Appendix A. Numerical method}

The equations of motion (2.1)-(2.4) are integrated by means of an eighth-order adaptable-step-size Runge-Kutta routine due to Dorman \& Price (see Hairer, Nørsett \& Wanner, p. 435 (1996)). Owing to the long time of most of the integrations involved, a natural question arises about the validity of the integration process. Previous work by Pullin \& Saffman (1991) has in fact showed some striking differences between using a fourth-order fixed-step-size Runge-Kutta scheme and a symplectic scheme. Nevertheless, it has to be pointed out that so far no direct comparison has been made between adaptable-step-size Runge-Kutta routines and symplectic ones. In particular, nowadays, a considerable number of high-order adaptable-step-size Runge-Kutta routines are available, which outperform (in terms of efficiency, speed, etc.) fourth-order fixed-step ones. For this purpose we compare the results of using fourth-(RK4) (Press et al. 1992) and eighth (RK8) order adaptable-step-size schemes to those of a fixed-step-size, two stage (SI2) fourth-order Gauss-Legendre implicit symplectic scheme, $\dagger$ similar to the one used by Pullin \& Saffman (1991) (described in the appendix of that article or in Sanz-Serna \& Calvo 1994). Concerning energy conservation, as shown in figure $25(a)$, while for both RK8 and SI2, the energy variations $|(H(t)-H(0)) / H(0)|$ show a similar non-increasing behaviour with time, with a maximum value of about $10^{-12}$, for RK4 there is a slow, but steady, increase

$\dagger$ As symplectic code we have used an implementation of code written by Leonid Kuznetsov (New York University). 
(a)
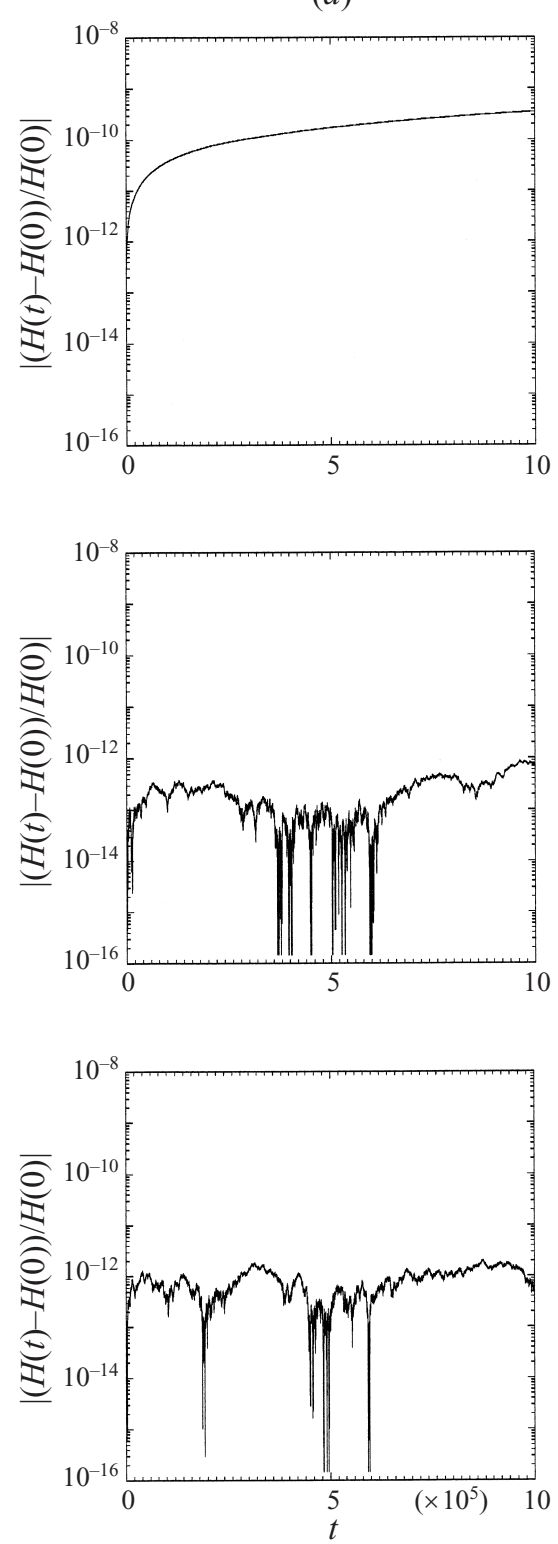

RK4

(b)

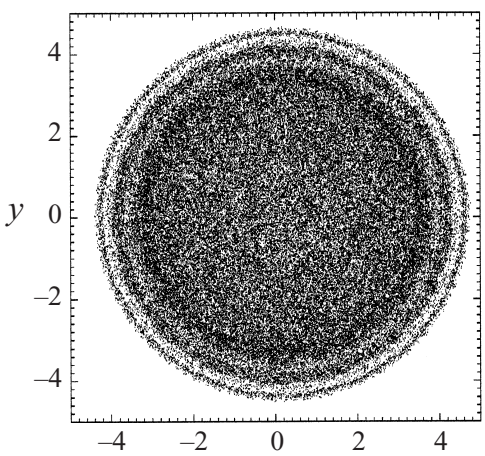

RK8

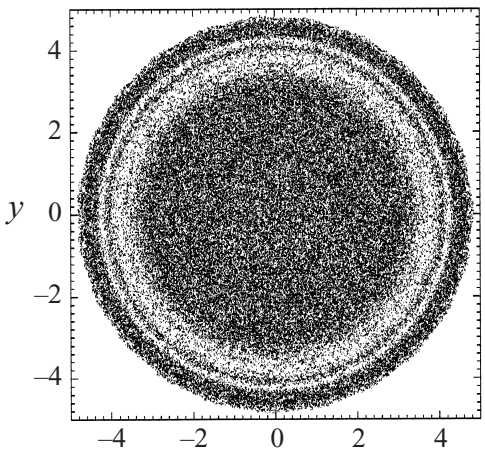

SI2

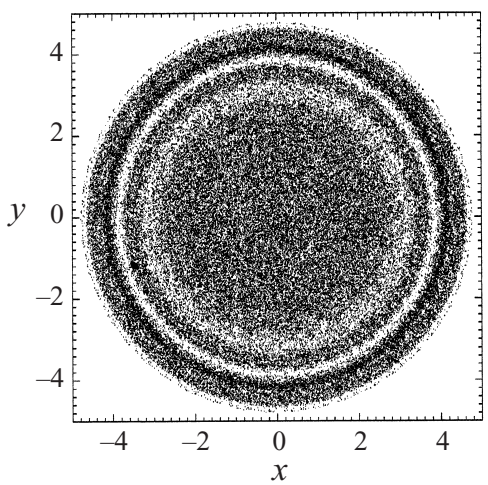

FIGURE 25. Comparison of results generated by adaptable-step-size Runge-Kutta routines, of fourth (RK4) and eighth (RK8) order (of accuracy of $10^{-12}$ ), and a second-order implicit Gauss-Legendre symplectic routine (SI2) of fixed step size $(\Delta T=0.00125)$. (a) Energy conservation of the vortex system. $(b)$ Trajectories of a tracer with initial conditions $(x(0), y(0))=(0.6,0)$. In all cases the vortex dynamics is chaotic $\left(\epsilon_{A}=0.5\right)$ and results were recorded for a time $T=10^{6}$.

of the energy variation. It has to be remarked that, despite these differences, after a time of $T=10^{6}$ the energy variation for RK4 is still quite small, of the order of $10^{-9}$.

Concerning 'morphological' differences, we have found similar features in using all three integration schemes. As shown in figure $25(b)$, in all three cases, the trajectory of a tracer advected by a chaotic vortex motion is preferentially trapped in annular 
orbits, as soon as it exits the central disk. The exact locations of the 'allowed' (darker regions in the figure) and the 'forbidden' (lighter annular regions in the figure) regions change with the routine, but this difference should not be important. In $\S 6$ we have in fact conjectured that the partition of the outer region into forbidden and allowed orbits is due to the presence of resonances, kicking the particle to another region of the phase space. What makes a resonance active is its strength, which could vary with time and make a region previously forbidden allowed at a later time. Since we are dealing with chaotic orbits, the different locations of the allowed and forbidden regions could simply correspond to two initially close orbits and therefore be a simple manifestation of chaos.

\section{Appendix B. FTLE analysis for a passive tracer in a one-point-vortex velocity field}

In $\S 4$ we have discussed two algorithms for estimating FTLEs. One is of general validity and evaluates the FTLE as the asymptotic slope of the curve $F\left(\boldsymbol{r}_{o}, t\right)=$ $\ln \left(\mathrm{d}\left(\boldsymbol{r}_{o}, t\right) / \mathrm{d}\left(\boldsymbol{r}_{o}, 0\right)\right)$, while the second, (4.8), gives a good representation of the system dynamics only for strictly hyperbolic systems. To illustrate this point further we consider a strictly non-hyperbolic dynamical system: a tracer in the velocity field of one point vortex. This system is integrable, so it is possible to compare numerical and analytical estimates for the Lyapunov exponent $\lambda\left(\boldsymbol{r}_{o}\right)$. The evolution equations for the tracer are

$$
\frac{\mathrm{d} x}{\mathrm{~d} t}=-\Omega y, \quad \frac{\mathrm{d} y}{\mathrm{~d} t}=+\Omega x,
$$

where $\Omega=K / 2 \pi R^{2}$ for vortex strength $K$. The radial distance $R$ is obviously a constant of motion and the tracer moves on circular orbits described by

$$
x=R \cos \left(\Omega t+\theta_{o}\right), \quad y=R \sin \left(\Omega t+\theta_{o}\right) .
$$

Two different orbits will be characterized by different radii, $R_{1}$ and $R_{2}$, and frequencies, $\Omega_{1}$ and $\Omega_{2}$, and the corresponding trajectory separation is a periodic function of time

$$
\mathrm{d}\left(\boldsymbol{r}_{o}, t\right)=\sqrt{R_{1}^{2}+R_{2}^{2}-2 R_{1} R_{2} \cos \left[\left(\Omega_{1}-\Omega_{2}\right) t\right]} .
$$

For every point $\boldsymbol{r}_{o}$ in space, the Lyapunov exponent is then found to be identically zero:

$$
\begin{aligned}
\lambda\left(\boldsymbol{r}_{o}\right) & =\lim _{t \rightarrow \infty} \lim _{R_{2} \rightarrow R_{1}} \frac{1}{t} \ln \frac{\mathrm{d}\left(\boldsymbol{r}_{o}, t\right)}{\mathrm{d}\left(\boldsymbol{r}_{o}, 0\right)} \\
& =\lim _{t \rightarrow \infty} \lim _{R_{2} \rightarrow R_{1}} \frac{1}{2 t} \ln \left(R_{1}^{2}+R_{2}^{2}-2 R_{1} R_{2} \cos \left[\left(\Omega_{1}-\Omega_{2}\right) t\right]\right)=0 .
\end{aligned}
$$

Using the first algorithm and computing FTLE as an asymptotic slope, we find $\lambda\left(\boldsymbol{r}_{o}, T\right) \rightarrow 0$ as $T \rightarrow \infty$. In contrast, evaluating the FTLE by means of the second algorithm, (4.8), we obtain

$$
\lambda\left(\boldsymbol{r}_{o}, T\right)=\frac{1}{T} \sum_{n=1}^{N} \lambda\left(t_{n}\right)^{+} \Delta \tau=\frac{1}{N \Delta \tau} N \lambda^{+} \Delta \tau=\lambda^{+},
$$

where $T=N \Delta \tau, \Delta \tau$ is the sampling time and $\lambda\left(t_{n}\right)^{+}=\lambda^{+}=k /\left(\sqrt{2} \pi R^{2}\right)$ is the 
maximum eigenvalue of the Jacobian matrix $\boldsymbol{J}=\left(\partial \boldsymbol{V} /\left.\partial \boldsymbol{x}\right|_{\boldsymbol{x}\left(t_{n}\right)}\right)$, i.e.

$$
J=\frac{k}{\pi R^{2}}\left(\begin{array}{cc}
\frac{x y}{R^{2}} & \frac{y^{2}}{R^{2}}-\frac{1}{2} \\
-\frac{x^{2}}{R^{2}}+\frac{1}{2} & -\frac{x y}{R^{2}}
\end{array}\right) .
$$

$\lambda\left(\boldsymbol{x}_{o}, T\right)$ is found to be a constant. Thus, using this second method, the FTLE would appear to be positive and, even in the limit $T \rightarrow \infty$, it would remain bounded away from zero, hinting at the existence of chaotic behaviour of the system, which we know to be false.

The apparent chaoticity indicated by the second algorithm comes from the fact that this method does not take account of angles in the direction of maximum stretching, which might give rise to a cancellation. Notice that this stretching-cancellation phenomenon is quite general and occurs whenever we have a potential flow with a region of closed orbits. This occurs because potential flows have a Jacobian of the form

$$
\mathbf{J}=\left(\begin{array}{rr}
a & b \\
c & -a
\end{array}\right) \text {. }
$$

It is easy to show that the eigenvalues $\lambda$ of $\boldsymbol{J}$ satisfy $\lambda^{2}=a^{2}+b c$, and, therefore, are always of opposite sign $\left(\lambda_{1}=-\lambda_{2}\right)$.

\section{REFERENCES}

Aref, H. 1982 Point vortex motions with a center of symmetry. Phys. Fluids 25, 2183.

AreF, H. 1984 Stirring by chaotic advection. J. Fluid Mech. 143, 1-21.

Aref, H. \& Pomphrey, N. 1982 Integrable and chaotic motions of four vortices. I. The case of identical vortices. Proc. R. Soc. Lond. A 380, 359-387.

ARnold, V. I. 1980 Mathematical Methods of Classical Mechanics. Springer.

Babiano, A., Boffetta, G., Provenzale, A. \& Vulpiani, A. 1994 Chaotic advection in point vortex models and two-dimensional turbulence. Phys. Fluids 6, 2465-2474.

Badi, R. \& Politi, A. 1987 Renyi dimensions from local expansion rates. Phys. Rev. A 35, 1288.

Bettin, G., Galgani, G., Giorgilli, A. \& Strelcyn, J. M. 1980 Lyapunov characteristic exponents for smooth dynamical systems and for Hamiltonian systems; a method for computing all of them. Mechanica, March, 21.

BoATto, S. 1996 Dynamics of a passive tracer in the velocity field of four identical point-vortices. $\mathrm{PhD}$ thesis, University of Chicago (June).

Carnevale, G. F., McWilliams, J. C., Pomeau, Y., Weiss, J. B. \& Young, W. R. 1991 Evolution of vortex statistics in two-dimensional turbulence. Phys. Rev. Lett. 66, 2735.

Cartwright, J. H. E. \& Piro, O. 1992 The dynamics of Runge-Kutta methods. Intl J. Bifurcation Chaos 2, 427-449.

Celletti, A. \& Falcolini, C. 1988 A remark on the KAM theorem applied to a four-vortex system. J. Statist. Phys. 52, 471-477.

Celletti, A. \& Chierchia, L. 1987 Rigorous estimates for a computer-assisted KAM theory. $J$. Math. Phys. 28, 2078-2086.

Chertkov, M., Falkovich, G., Kolokolov I. \& Lebedev, V. 1995 Statistics of a passive scalar advected by a large-scale two-dimensional velocity field: Analytic solution. Phys. Rev. E 51, $5609-5627$.

Chorin, A. J. \& Marsden, J. E. 1993 A Mathematical Introduction to Fluid Mechanics. Springer.

Crisanti, A., Falcioni, M., Vulpiani, A. \& Paladin, G. 1991 Lagrangian chaos: transport, mixing and diffusion in fluids. Rivista del Nuovo Cimento 14 (12), 1-79.

Hairer, E., Nørsett, S. P. \& Warner, G. 1996 Solving Ordinary Differential Equations. Springer.

Horita, T., Hata, H., Ishizaki, R. \& MoRi, H. 1990 Long-time correlations and expansion-rate spectra of chaos in Hamiltonian systems. Prog. Theor. Phys. 83, 1065-1070. 
Kirchioff, G. R. 1876 Vorlesungen über mathematische. Physik. Mechanik, Leipzig, Teubner. Lichtenberg, A. J. \& Lieberman, A. M. 1992 Regular and Chaotic Dynamics. Springer.

Mackay, R. S., Meiss, J. D. \& Percival, I. C. 1987 Resonances in area-preserving maps. Physica 27D, 1.

Marsden, J. E. \& Scheurle, J. 1995 Pattern evocation and geometric phases in mechanical systems with symmetry. Dyn. Stab. Systems 10, 315-338.

Mather, J. N. 1984 Non-existence of invariant circles. Ergod. Theor. Dyn. Syst. 4, 301-309.

MCLachlan, R. I. \& Atela, P. 1992 The accuracy of symplectic integrators. Nonlinearity 5, 541-562.

McWilliams, J. C. 1984 The emergence of isolated coherent vortices in turbulent flow. J. Fluid Mech. 146, 21-43.

Montroll, E. \& Schlesinger, M. 1984 In Non-Equilibrium Phenomena II: From Stochastics to Hydrodynamics (ed. J. Leibowitz \& E. Montroll). North-Holland.

Morbidelli, A. \& Guzzo, M. 1996 The Nekhoroshev theorem and the asteroid belt dynamical system. Celest. Mech. Dyn. Astron. 65, 107-136.

Muzzio, F. J., Swanson, P. D. \& OtTino, J. M. 1991 The statistics of stretching and stirring in chaotic flows. Phys. Fluids A 3 (5), 822-834.

Novikov, E. A. 1976 Dynamics and statistics of a system of vortices. Sov. Phys. JETP 41, 937-943.

OSELEDEC, V. I. 1968 A multiplicative ergodic theorem. Lyapunov characteristic numbers for dynamical systems. Trans. Moscow Math. Soc. 19, 197.

Otт, E. 1993 Chaos in Dynamical Systems. Cambridge University Press.

Percival, I. C. 1979 In Nonlinear Dynamics and Beam-Beam Interaction (ed. M. Month \& J. C. Herrera). AIP Conference Proceedings no. 57, pp. 302. American Institute of Physics

Pierrehumbert, R. T. $1991 b$ Large-scale horizontal mixing in planetary atmospheres. Phys. Fluids A 3, 1250 .

Pierrehumbert, R. T. \& YANG, H. 1993 Global chaotic mixing on isentropic surfaces. J. Atmos. Sci. 50, 2462-2480.

Press, W. H., Flannery, B. P., Teukolsky, S. A. \& Vetterling, W. T. 1992 Numerical Recipes. Cambridge University Press.

Pullin, D. I. \& Saffman, P. G. 1991 Long-time symplectic integration: the example of four-vortex motion. Proc. R. Soc. Lond. A 432, 481-494.

Rom-Kedar, V. 1993 The topological approximation method. In Transport Chaos \& Plasma Physics, p. 1. IMT, Marseilles.

Sanz-Serna, J. M. \& Calvo, M. P. 1994 Numerical Hamiltonian Problems. Chapman \& Hall.

Shraiman, B. I. \& Siggia, E. D. 1994 Lagrangian path integrals and fluctuations in random flow. Phys. Rev. E 49, 2912-2927.

Simó, C. 1994 Averaging under fast quasi-periodic forcing. In Hamiltonian Mechanics (Toruń, 1993). NATO Adv. Sci. Inst. B: Phys., vol. 331, pp. 13-34.

Sommeria, J., Meyers, S. D. \& Swinney, H. L. 1988 Laboratory model of a planetary eastward jet. Nature 337, 58-61.

Wiggins, S. 1991 Chaotic Transport in Dynamical Systems. Springer.

Wiggins, S. \& Malhotra, N. 1998 Geometric structures, lobe dynamics, and Lagrangian transport in flows with aperiodic time-dependence, with applications to Rossby wave flow. J. Nonlinear Sci. 8, 401-456. 